\title{
Protein Crowding within the Postsynaptic Density Can Impede the Escape of Membrane Proteins
}

\author{
Tuo P. Li, ${ }^{1}$ Yu Song, ${ }^{2}$ Harold D. MacGillavry, ${ }^{1}$ Thomas A. Blanpied, ${ }^{1}$ and Sridhar Raghavachari ${ }^{3}$ \\ ${ }^{1}$ Department of Physiology and Program in Neuroscience, University of Maryland School of Medicine, Baltimore, Maryland 21201, ${ }^{2}$ Department of Physics, \\ Duke University, Durham, North Carolina 27708, and ${ }^{3}$ Department of Neurobiology, Duke University Medical Center, Durham, North Carolina 27710
}

Mechanisms regulating lateral diffusion and positioning of glutamate receptors within the postsynaptic density (PSD) determine excitatory synaptic strength. Scaffold proteins in the PSD are abundant receptor binding partners, yet electron microscopy suggests that the PSD is highly crowded, potentially restricting the diffusion of receptors regardless of binding. However, the contribution of macromolecular crowding to receptor retention remains poorly understood. We combined experimental and computational approaches to test the effect of synaptic crowding on receptor movement and positioning in Sprague Dawley rat hippocampal neurons. We modeled AMPA receptor diffusion in synapses where the distribution of scaffold proteins was determined from photoactivated localization microscopy experiments, and receptorscaffold association and dissociation rates were adjusted to fit single-molecule tracking and fluorescence recovery measurements. Simulations predicted that variation of receptor size strongly influences the fractional synaptic area the receptor may traverse, and the proportion that may exchange in and out of the synapse. To test the model experimentally, we designed a set of novel transmembrane (TM) probes. A single-pass TM protein with one PDZ binding motif concentrated in the synapse as do AMPARs yet was more mobile there than the much larger AMPAR. Furthermore, either the single binding motif or an increase in cytoplasmic bulk through addition of a single GFP slowed synaptic movement of a small TM protein. These results suggest that both crowding and binding limit escape of AMPARs from the synapse. Moreover, tight protein packing within the PSD may modulate the synaptic dwell time of many TM proteins important for synaptic function.

Key words: FRAP; GFP imaging; inducible dimerization; single-molecule tracking; stochastic modeling; uPAINT

Significance Statement

Small alterations to the distribution within synapses of key transmembrane proteins, such as receptors, can dramatically change synaptic strength. Indeed, many diseases are thought to unbalance neural circuit function in this manner. Processes that regulate this in healthy synapses are unclear, however. By combining computer simulations with imaging methods that examined protein dynamics at multiple scales in space and time, we showed that both steric effects and protein-protein binding each regulate the mobility of receptors in the synapse. Our findings extend our knowledge of the synapse as a crowded environment that counteracts molecular diffusion, and support the idea that both molecular collisions and biochemical binding can be involved in the regulation of neural circuit performance.

\section{Introduction}

Synaptic strength is modulated by both the density and positioning of AMPARs within the synapse (Xie et al., 1997; Franks et al.,

Received Aug. 21, 2015; revised Feb. 17, 2016; accepted Feb. 19, 2016.

Author contributions: T.P.L., Y.S., H.D.M., T.A.B., and S.R. designed research; T.P.L., Y.S., H.D.M., and S.R. performed research; T.P.L. and S.R. contributed unpublished reagents/analytictools; T.P.L., Y.S., and S.R. analyzed data; T.P.L., T.A.B., and S.R. wrote the paper.

This work was supported by the National Institutes of Health Grant F30MH102891 to T.P.L., Grant R01MH080046 to T.A.B., and Grant R01MH096376 to S.R., the Kahlert Foundation to T.A.B., and the Katherine D. and Theodore J. Karski Foundation to T.A.B. We thank Hsiangmin Lu, Scott Thompson, Paul Welling, Bruce Krueger, Bradley Alger, Tom Abrams, and members of the Thompson laboratory and the T.A.B. laboratory for helpful discussions; and Tamar Davis and Minerva Contreras for help with cultures.

The authors declare no competing financial interests.

Correspondence should be addressed to either of the following: Dr. Sridhar Raghavachari, Department of Neurobiology, Duke University Medical Center, Durham, NC 27710, E-mail: sraghava@nsf.gov; or Dr. Thomas A. Blan-
2003; Raghavachari and Lisman, 2004; Lisman et al., 2007; Kessels and Malinow, 2009), so determining the mechanisms that establish their synaptic entry, exit, and distribution is of great interest. We argue here that key mechanisms controlling AMPAR dynamics in the synapse are likely also to apply to the many other transmembrane (TM) proteins that establish synapse structure and function.

pied, Department of Physiology and Program in Neuroscience, University of Maryland School of Medicine, Baltimore, MD 21201, E-mail: tblanpied@som.umaryland.edu.

H.D. MacGillavry's present address: Cell Biology, Department of Biology, Faculty of Science, Utrecht University, 3584 CH Utrecht, The Netherlands.

S. Raghavachari's present address: Department of Biology, University of Maryland, College Park, MD 20742.

DOI:10.1523/JNEUROSCI.3154-15.2016

Copyright $\odot 2016$ the authors $\quad 0270-6474 / 16 / 364276-20 \$ 15.00 / 0$ 
Receptors and other critical synaptic TM proteins are highly mobile in the dendritic plasma membrane (Meier et al., 2001; Borgdorff and Choquet, 2002; Sergé et al., 2002; Breillat et al., 2007; Biermann et al., 2014). This mobility is generally sufficient to permit TM proteins to explore large expanses of the dendrite on the time scale of minutes, to enter and exit spines efficiently, and perhaps most importantly, to encounter numerous synapses where they may be incorporated (Giannone et al., 2010; Masson et al., 2014). It is now well established in particular that AMPARs enter and exit the synapse via lateral diffusion (Borgdorff and Choquet, 2002; Heine et al., 2008). Given this, it is widely expected that synapses maintain steady-state enrichment of receptors by presenting binding sites that slow receptor diffusion (Gerrow and Triller, 2010). Indeed, a large fraction of AMPARs within the synapse are highly constrained and nearly immobile (Bats et al., 2007; Ehlers et al., 2007; Kerr and Blanpied, 2012; Choquet and Triller, 2013). In addition, PDZ-mediated interactions between transmembrane AMPAR regulatory proteins (e.g., Stargazin) and the major synaptic scaffolding protein PSD-95 (postsynaptic density-95) play a key role in reducing the lateral mobility of AMPARs at synapses (Bats et al., 2007). However, peptides that acutely disrupt their binding decrease synaptic currents by only $20 \%-50 \%$ (Sainlos et al., 2011). This suggests that Stargazin-PSD-95 binding cannot solely determine AMPAR number and mobility at the synapse.

Macromolecular crowding could be a key mechanism that complements receptor-scaffold interactions to regulate the mobility of TM proteins in the synapse. The density of proteins in the PSD is extremely high near the cell membrane (Husi et al., 2000; Sheng and Hoogenraad, 2007; Burette et al., 2012), likely imposing a series of physical barriers to hinder lateral diffusion of TM proteins (Trimble and Grinstein, 2015). Indeed, diffusion of extracellularly labeled lipids in the synapse is affected by the extent of the protein bulk used for labeling (Renner et al., 2009b), suggesting a role for excluded volume interactions in regulating molecular mobility. However, because lipids can preferentially sample membrane regions not accessible by most TM proteins and extracellularly labeled lipids are most likely not influenced by the cytosolic environment, it is unclear whether these observations apply in general to TM proteins containing extended intracellular domains. Computational modeling of receptor diffusion in crowded PSDs showed that, even in PSDs devoid of receptorbinding proteins, synaptic receptors could be retained for minutes or even hours merely because hindrance by transmembrane and membrane-proximal proteins reduced their mobility (Santamaria et al., 2010). Furthermore, proteins in the PSD are not just densely packed but are heterogeneously distributed with notable clustering (MacGillavry et al., 2013; Nair et al., 2013) that may accentuate steric effects. Despite these observations, the influence of intracellular protein bulk in determining synaptic mobility of TM proteins has not been directly tested.

To address this, we distilled a receptor to its barest essence, a single-pass transmembrane domain and a cytosolic tail that can bind to PSD-95. This probe accumulates in synapses yet is dramatically smaller than AMPARs, allowing us to manipulate protein bulk and binding capacity independently. Combining dynamic imaging with theoretical modeling, we show that mobility of these probes within the PSD is affected in a size-dependent manner by both steric effects and biochemical binding. Our results suggest that steric effects may regulate the mobility of a large diversity of synaptic TM proteins, with implications for synaptic transmission and plasticity.
Table 1. Simulation parameters

\begin{tabular}{|c|c|c|}
\hline & Value & Study \\
\hline PSD-95 radius & $2.5 \mathrm{~nm}$ & Chen et al. (2008) \\
\hline NMDAR radius & $8 \mathrm{~nm}$ & Chen et al. (2008) \\
\hline Adhesion molecules, radii & $3-8 \mathrm{~nm}$ & This work $^{a}$ \\
\hline Other immobile proteins, radii & $5-10 \mathrm{~nm}$ & This work $^{a}$ \\
\hline NMDAR number & 20 & Cheng et al. (2006) \\
\hline Adhesion molecule number & 48 & Cheng et al. (2006) \\
\hline Other immobile protein number & 15 & Cheng et al. (2006) \\
\hline Diameter of PSD & $140-400 \mathrm{~nm}$ & MacGillavry et al. (2013) \\
\hline AMPAR number within a synapse & 100 & Matsuzaki et al. (2001) \\
\hline AMPAR diffusion constant & $0.2 \mu \mathrm{m}^{2} / \mathrm{s}$ & Ehlers et al. (2007) \\
\hline
\end{tabular}

${ }^{a}$ Radii of assumed unstructured conformations were deduced from total counts of amino acids based on the radius of gyration estimates of peptide chains by Kohn et al. (2004).

\section{Materials and Methods}

\section{For simulations}

Synapse geometry. We approximated the PSDs as convex hulls, the boundaries of which were extracted from the PSD-95 coordinates directly measured with PALM imaging (MacGillavry et al., 2013). The PSD area ranged from 0.02 to $0.53 \mu \mathrm{m}^{2}(n=100)$. The density of PSD-95 inside each PSD was kept to $\sim 3000 / \mu \mathrm{m}^{2}$ (Chen et al., 2011). The PSD-95 molecules acted as obstacles to receptor diffusion. Adhesion molecules, NMDA type glutamate receptors, and other proteins were also added to the system (Table 1).

Two-dimensional percolation for receptors within PSD. We adapted methods from computational geometry that were recently used to study percolation (Saxton, 2010). Briefly, transmembrane and juxtamembrane proteins, such as PSD-95, were modeled as disks distributed on a plane. Given the set of points representing the centers of the disks, the Voronoi diagram divides the plane into polygons, each containing a single point. The dual of the Voronoi diagram, known as the Delaunay triangulation, is a set of triangles that tiles the plane, with the vertices at the different points and the edge lengths, $D_{i j}$ representing the distance between the points $i$ and $j$. The region of polygon belonging to one point is closer to that point than any other point. If a tracer's diameter, $d_{t r a c}$ is smaller than $D_{i j}-r_{i}-r_{j}$ (the edge length minus the radii of the two points), then the tracer can move across the edge between the two particles. The edge connecting the particle centers $i$ and $j$ is then labeled a "conducting edge." Thus, given a distribution of differently sized disks on a plane, all the conducting edges can be identified for a given tracer diameter. If a tracer can enter, traverse, and exit a distribution of disks at distinct points, then a percolation path exists for those particles.

To investigate the effects of PSD molecular crowding and receptorscaffolding binding on AMPAR concentration, we considered the $2 \mathrm{D}$ movement of a tracer particle in the presence of a set of effectively immobile molecules, such as PSD-95, NMDA receptors, and adhesion molecules. We first placed PSD-95 molecules based on coordinates estimated by PALM experiments (MacGillavry et al., 2013) and then randomly placed a set of transmembrane proteins without spatial overlap within the PSD border. These include AMPA receptors, NMDA receptors, adhesion molecules, and other ion channels; their numbers were prescribed based on density estimated by EM and mass spectrometry experiments (Sheng and Hoogenraad, 2007). NMDARs and the initial locations of AMPARs were distributed relative to PSD-95 by matching the number distribution of PSD-95 neighbors as determined from measured twocolor localizations (MacGillavry et al., 2013) while also ensuring that individual molecules did not overlap. Although the other non-PSD-95 proteins were randomly placed, the constraint of nonoverlapping spatial arrangement yielded nonrandom distributions in which most of these proteins were more likely to be excluded from the highly dense PSD-95 regions and thus relatively enriched toward the edge of the synapse. Nevertheless, the occupied volume of these molecules is far less than that of the receptors and PSD-95 combined and would therefore have a smaller effect on receptor mobility. We set the radius of PSD-95 to be 2.5 $\mathrm{nm}$ as estimated by EM tomography (Chen et al., 2008). We set the extracellular and transmembrane radii of the AMPAR complex to be 8 
and $5 \mathrm{~nm}$, respectively, as estimated by single-particle EM (Nakagawa et al., 2005). To model the yet to be crystallized intracellular bulk of the AMPAR complex, we used estimates from single-particle EM of GluK2 intracellular domain and a mathematical estimate of Stargazin C-tail size based on its residue count. (Schauder et al. (2013) showed that the putative intracellular domain of a full-length GluK2 receptor is $\sim 10 \mathrm{~nm}$ in width and $5 \mathrm{~nm}$ in height. TARP proteins have not been crystallized, but the intracellular tail ( $N=\sim 120$ amino acids) of the most studied TARP representative Stargazin is estimated to be $\sim 3 \mathrm{~nm}$ in radius assuming an unstructured globule, as calculated from the radius of gyration of a random polymer $R_{g}^{2} \approx \frac{1}{6} N^{6 / 5} b^{2}$ (Kohn et al., 2004), where $N$ is the number of amino acids and $b$ is a constant that is a function of the persistent length of the polymer. Given the residue count of GluK2 cytoplasmic portion is intermediate between that of GluA1 and that of GluA2 and that full AMPARs can contain anywhere from 1 to 4 Stargazin subunits (Hastie et al., 2013), we conservatively modeled the intracellular radius of the AMPAR complex to be $8 \mathrm{~nm}$. The radii of adhesion molecules were taken from a random distribution ranging from 3 to $8 \mathrm{~nm}$. The sizes of other immobile proteins (e.g., ion channels) were taken from a random distribution ranging from 5 to $10 \mathrm{~nm}$. The Voronoi diagram was computed in MATLAB (The MathWorks). Poisson distributions of PSD-95 were generated by first drawing a convex hull to mark the boundary around the measured positions of PSD-95 and then randomly placing the particles within the hull while ensuring that two randomly placed particles did not overlap.

The percolation paths were found based on the diameter of the diffusing tracer and the boundaries between the mentioned molecules. We evaluated the conducting area numerically as the area of the Voronoi regions that had at least one conducting edge for different measured PSD configurations used in the percolation calculations. We calculated the conducting area fraction as the ratio of area with conducting paths divided by total PSD area at different tracer sizes.

Simulating AMPAR diffusion and binding. To simulate AMPAR diffusion within the PSD and the surrounding extrasynaptic space, we used Monte Carlo simulations of Brownian dynamics with a discretization time step of $2 \mu \mathrm{s}$. For sufficiently small time interval $\Delta t$, each time step is simulated based on the following equations:

$$
\begin{aligned}
& \Delta x=\sqrt{2 D \Delta t} \xi_{x} \\
& \Delta y=\sqrt{2 D \Delta t} \xi_{y}
\end{aligned}
$$

where $\xi_{x}, \xi_{y}$ are Gaussian-distributed white noise. The AMPAR diffusion coefficient outside synapses was chosen to be in the range of $0.2 \mu \mathrm{m}^{2} / \mathrm{s}$, based on single-particle tracking data by Ehlers et al. (2007) and current data. The diffusion space was chosen as a circular domain of 400 $\mathrm{nm}$ radius with a central region occupied by the PSD. At each time step, trial position $(x, y)$ coordinates of AMPARs were incremented by the distances $(\Delta x, \Delta y)$; however, whether a receptor could diffuse to the new position depended on several rules: (1) whether it bound to PSD-95 upon collision, and (2) whether it collided with nearby immobile obstacles. Once it bound to PSD-95, the particle stopped lateral diffusion but underwent rotational diffusion, which determined its subsequent position of dissociation. A bound receptor molecule could dissociate from a PSD-95 molecule according to a first-order reaction. Receptors underwent free diffusion in extrasynaptic space, which was treated as devoid of any obstacles. Although the PSD is a complex network of scaffold proteins, we pooled all binary and unitary reactions into two first-order kinetic rates, $\mathrm{K}_{\text {on }}$ and $\mathrm{K}_{\text {off }}$. Together, this model of receptor dynamics accounts for (1) association with scaffolding molecules, (2) dissociation from scaffolding molecules, (3) collision with obstacles when diffusing, and (4) free diffusion outside the PSD. Given that scaffold molecules at the synapse are in excess compared with the number of AMPARs (Sheng and Kim, 2011), we kept $K_{\text {on }}$ constant. The dissociation rate $K_{\text {off }}$ was taken as the inverse of mean dwell time of AMPARs within postsynaptic density. Simulation parameters were chosen based on estimates of other studies (Table 1).
Simulating full and partial synapse FRAP. Diffusible particles were labeled as fluorescent and randomly placed in the PSD and in a bigger domain (circle R) surrounding the PSD. The circle R radius was set to 400 $\mathrm{nm}$. Particles were allowed to reach before photobleaching. In case of full PSD bleaching, all particles within the convex hull of the PSD were set to be nonfluorescent, whereas the ones outside remained marked as fluorescent. The number of fluorescent particles within the convex hull of the PSD were recorded as a function of time to calculate the FRAP curve. In case of partial synapse FRAP, particles within half the convex hull of the PSD were considered as bleached. While in simulations in Figures 1 and 2 , the total number of receptors was fixed, FRAP simulations needed to account for the exchange of receptors between the synaptic/perisynaptic region and the rest of the spine. To account for this exchange, a third parameter, $\mathrm{K}_{\text {flip }}$, was introduced. $\mathrm{K}_{\text {flip }}$ was defined as the rate at which photobleached receptors get replaced by fluorescent receptors when they hit the outside boundary circle $\mathrm{R}$. We tried different values of $\mathrm{K}_{\text {on }}, \mathrm{K}_{\text {off }}$, and $\mathrm{K}_{\text {flip }}$ to fit experimental FRAP recovery curves, and we chose the values that fit the experimental FRAP with the least squared error.

\section{For experiments}

Neuron culture and transfection. Dissociated hippocampal neuron cultures were prepared from embryonic day 18 rats of both sexes as previously described (Frost et al., 2010). For PALM and uPAINT experiments, cells were plated onto coverslips that were cleaned and coated as reported by MacGillavry et al. (2013). Cells were grown 2-3 weeks in culture and transfected $36-48 \mathrm{~h}$ before experiments (unless stated otherwise). Individual coverslips were transfected with $0.5-1.0 \mu \mathrm{g}$ of cDNA for each expression construct using Lipofectamine 2000 (Invitrogen).

Expression constructs. cDNAs were obtained or produced as follows (with original sources): superecliptic pHluorin (SEP)-GluA2 (R. Huganir); mEos3-GluA2 and PSD-95-mEos2 (MacGillavry et al., 2013). SEP-TM and mEos3-TM were constructed by subcloning SEP from SEPGluA2 and mEos3.1 from mEos3.1-N1 (S. McKinney), respectively, into the pDisplay vector (Invitrogen) at XmaI-SacII; SEP-TM-Bind and mEos3-TM-Bind were constructed by subcloning TM-StgCtail into SEP-TM and mEos3-TM at SacII-XhoI. TM-StgCtail was bought as a single dsDNA segment (gBlocks Gene Fragments, Integrated DNA Technologies), in which the TM was derived from the pDisplay vector, and the StgCtail derived from residues D203-V323 of Stargazin peptide sequence (UniProt ID O88602); multiple cloning sites flanking the TM-StgCtail were introduced to facilitate further customization: added features include AgeI and MluI upstream, as well as MluI and a stop codon downstream. Homerlc-mCh was prepared by subcloning Homerlc from Homer1c-GFP (P. Worley) to NheI-AgeI sites of mCh-N1 (R.Y. Tsien).

SEP-TM-Cerulean and mEos3-TM-Cerulean were made by removing StgCtail from SEP-TM-Cerulean-StgCtail and mEos3-TM-CeruleanStgCtail using MluI-mediated restriction and self-ligation. SEP/mEos3TM-Cerulean-StgCtail was made by PCR extracting mCerulean3 from mCerulean3-C1 (M.A. Rizzo) and subcloning into SEP/mEos3-TMStgCtail at AgeI. XbaI and NheI were added immediately upstream and downstream of mCerulean3, respectively, during PCR extraction for two purposes: (1) to facilitate verification of the correction orientation of insertion; and (2) to facilitate addition of multiple mCerulean3's in tandem as XbaI and NheI have overlapping sticky ends; thus, an XbaI-NheI-digested mCerulean 3 can be cloned into either XbaI or NheI site of a plasmid that contains both, which can be done multiple times without requiring additional restriction sites (adapted from iDimerize protocol of Clontech). However, SEP-TM-Cerulean-StgCtail and SEP-TM fused to multiple mCerulean3's on the intracellular domain, when expressed in neurons, did not show detectable fluorescence under neutral $\mathrm{pH}$ but fluoresced in ammonium-chloride-based alkaline bath (Park et al., 2006), indicating that the constructs do not traffic to the surface plasma membrane.

To generate SEP-FKBP-TM-Bind/Nonbind, FKBP was inserted into SacII site of SEP-TM-Bind/Nonbind. To generate TagBFP-FRB-TMBind/Nonbind, mTagBFP2-FRB was substituted for the SEP domain in SEP-TM-Bind/Nonbind between restriction sites XmaI and SacII. FKBP and $\mathrm{mTagBFP} 2-\mathrm{FRB}$, flanked by the appropriate restriction enzyme recognition sites, were purchased as gBlock dsDNA segments (IDT). The mTagBFP2 sequence was derived from pBAD-mTagBFP2, a kind gift 
from Vladislav Verkhusha (Addgene plasmid \#34632). The monomeric $\mathrm{FKBP}$ and $\mathrm{FRB}$ sequences were derived from $\mathrm{pC}_{4} \mathrm{M}-\mathrm{F} 2 \mathrm{E}$ and $\mathrm{pC}_{4}-\mathrm{R}_{\mathrm{H}} \mathrm{E}$, respectively (Ariad Pharmaceuticals), now available as pHet-Meml and pHet-1 (Clontech). All constructs were confirmed by sequencing.

Live-cell imaging conditions. Cells expressing the indicated constructs were imaged in extracellular imaging solution containing the following (in mM): $120 \mathrm{NaCl}, 3 \mathrm{KCl}, 2 \mathrm{CaCl}_{2}, 2 \mathrm{MgCl}_{2}, 10$ glucose, 10 HEPES, $\mathrm{pH}$ adjusted to 7.35 with $1 \mathrm{~N} \mathrm{NaOH}$. Cells remained at $25^{\circ} \mathrm{C}$ for no more than $1 \mathrm{~h}$ per imaging session. In experiments where rapalog (AP21967 or A/C Heterodimerizer, Clontech) was applied, $100 \mu \mathrm{l}$ of $10 \times$ treatment solution was added to the $0.9 \mathrm{ml}$ bath. Rapalog was supplied in ethanol; it was diluted to $10 \mu \mathrm{M}$ in the extracellular imaging solution from a $500 \times$ stock solution. Thus, each application of rapalog resulted in a final concentration of $0.2 \%$ ethanol.

Fluorescence recovery for up to 11 min after photobleaching. Short-term FRAP experiments were performed on a spinning disk confocal system using software acquisition control by iQ (Andor Technology) similar to what has been previously described (Lu et al., 2014); however, it was modified to use a sCMOS camera (Zyla, Andor Technology) and a computer-steered, galvo-scanning laser delivery system (FRAPPA, Andor Technology). Imaging was performed with a $100 \times 1.45$ numerical aperture (NA) oil-immersion objective, yielding a final effective pixel size of $65 \mathrm{~nm}$. For recovery up to $140 \mathrm{~s}$ after photobleaching, images were single optical sections acquired every $2 \mathrm{~s}$ for 40 frames ( 20 before and 20 after bleaching) and subsequently every $5 \mathrm{~s}$ for 20 frames. $Z$ stability was maintained by ZDC2 (Olympus) feedback positioning system. For recovery of $11 \mathrm{~min}$ after full and partial synapse photobleaching, images were 5 -section $Z$-stacks ( $0.4 \mu \mathrm{m} /$ section $)$ acquired four times at variable delays after bleaching ( $5 \mathrm{~s}, 1 \mathrm{~min} 5 \mathrm{~s}, 6 \mathrm{~min} 5 \mathrm{~s}$, and $11 \mathrm{~min} 5 \mathrm{~s}$ ).

Photobleaching of synaptic SEP-tagged proteins was achieved via the FRAPPA unit using $405 \mathrm{~nm}$ laser excitation ( $488 \mathrm{~nm}$ laser was avoided as it bleached both SEP and mCherry fluorescence regardless of bleaching power, dwell time, or iterations). Bleaching was executed in all cases with $60 \mu$ s dwell time, 2 iterations at $40 \%-50 \%$ of the available $405 \mathrm{~nm}$ laser power. Bleaching parameters were adjusted at the beginning of each experiment to minimize $\mathrm{mCherry}$ bleaching. Time $=0$ image acquisition followed directly after photobleaching. For full-synapse photobleaching, a rectangular bleaching region was placed around an entire synapse or spine. In contrast, for partial synapse photobleaching, a small rectangular region was placed near the edge of synapses. This resulted in $67 \pm 2 \%$ bleached for the half of the synapse close to the bleaching spot and $40 \pm$ $4 \%$ bleached for the opposite half in the case of SEP-GluA2; $67 \pm 2 \%$ for half targeted for bleaching and $36 \pm 4 \%$ for the opposite half in the case of SEP-TM-Bind. On average, $50 \pm 3 \%$ of the total synaptic SEP fluorescence was lost during partial synapse bleaching. Each experiment contained some synapses that were targeted for partial synapse photobleaching, whereas others were fully bleached.

Fluorescence recovery for $52 \mathrm{~min}$ after photobleaching. Longer recordings of FRAP to measure the near-equilibrium states were performed on a Zeiss LSM710 laser scanning confocal system using a 20×/1.0 PlanApochromat water-immersion objective and software acquisition control by Zen. SEP was excited with a $488 \mathrm{~nm}$ Ar ion laser, and mCherry with a $561 \mathrm{~nm}$ DPSS laser. Selected Homer 1c-mCherry containing spines were bleached with 2 scans of the $488 \mathrm{~nm}$ laser at elevated power, which achieved $50 \%-70 \%$ reduction in synaptic SEP fluorescence with minimal bleaching of mCherry fluorescence. Images were 5-section $Z$-stacks (1.0 $\mu \mathrm{m} /$ section) acquired 7 times at variable delays after bleaching $(1,2,7$, $12,22,32$, and $52 \mathrm{~min}$ ). Pinhole size was set to $2-3$ Airy units, and a $4 \times$ optical zoom was used, yielding a pixel size of $104 \mathrm{~nm}$.

FRAP image analysis. Image processing and analysis was performed in ImageJ after export from iQ or Zen. Values measured in ImageJ were output to Prism (GraphPad Software), in which statistics and graphing were done. Image $z$-stacks at all time points were maximally projected in the $z$ dimension. Values measured in ImageJ were backgroundsubtracted before analysis. Baseline fluorescence intensity was normalized to 1.0, and FRAP recovery was calculated as the fluorescence increase between $t=0$ (immediately after SEP photobleaching) and the indicated time point. Rectangular regions were drawn to enclose individual spines or synapses while excluding the parent dendrite and minimizing back- ground contribution. In partial synapse photobleaching experiments, the SEP intensity of 9-15 randomly selected spines in each field not targeted for bleaching were quantified at each time point; in longer recordings of FRAP, two spines not targeted for bleaching were randomly selected. The SEP signal in unbleached spines served as a control for the bleaching that occurred during imaging acquisition. The recovery curves of the bleached spines were normalized to a linear fit of the average intensity of the unbleached spines over time.

For full synaptic photobleaching, a single region was drawn around the entire synapse using the Homer1c-mCherry image. Averaged fluorescence intensity of SEP-tagged protein was quantified at each time. For partial synaptic photobleaching, two regions were drawn. The bleached region was drawn based on where the original bleaching region of interest was targeted. An additional control region of similar size was placed on the opposite side of the synapse. StackRegJ (Jay Unruh, Stowers Institute for Medical Research, Kansas City, MO), a plugin of ImageJ, was used to correct for $X Y$ drift. StackRegJ aligns the mCherry channel time lapse based on the first time point in most cases, and then applies the same transformation to the SEP channel.

Synapses that were improperly bleached were discarded from further analysis. Synapses targeted for partial photobleaching could be improperly bleached for several reasons. For example, a single-pixel offset between the bleaching laser and the synapse before bleaching occurs can result in overbleaching or underbleaching. In addition, synapses can morph so much within a few minutes after photobleaching that the bleached spot cannot be relocated reliably. To avoid these, we excluded the following cases from further analysis: (1) synapses of which partial bleaching regions were $<60 \%$ bleached with respect to baseline fluorescence intensity; (2) synapses targeted for partial photobleaching that inadvertently resulted in decreased fluorescence over $>3 / 4$ of the synaptic area; and (3) synapses of which morphology changed so considerably within the $11 \mathrm{~min}$ experiment that analysis regions could not be repositioned with confidence. Using these criteria, 30 of 61 synapses targeted for partial photobleaching were excluded from further analysis.

We measured the areas of individual synapses by counting the image pixels of the Homer1c-mCherry cluster after thresholding the background-subtracted image at half the peak fluorescence intensity of the PSD. We measured spine enrichment of each SEP-tagged protein by first drawing a rectangular region to enclose the entire spine head with minimal contribution from the background, and then drawing a polygonal region $(\sim 3 \times$ the width of the spine region) to enclose the parent dendritic segment immediately near the spine. The dendritic region was occasionally offset laterally to avoid dendritic hot spots of Homer1cmCherry. SEP and Homer1c-mCherry (or TagBFP-FRB-TM-Bind) fluorescence in spines and dendrites was measured as averaged green and red (or blue) fluorescence after background subtraction. Spine enrichment was defined as $\left(G_{\text {Spine }} / R_{\text {Spine }}\right) /\left(G_{\text {Dendrite }} / R_{\text {Dendrite }}\right)$, where $G_{\text {Spine }}$ and $G_{\text {Dendrite }}$ represent the spine and dendritic SEP fluorescence, and $R_{\text {Spine }}$ and $R_{\text {Dendrite }}$ the spine and dendritic mCherry (or TagBFP) fluorescence. This method was used as a relative, not absolute, means of comparing enrichment levels.

Single-molecule tracking (smt). SmtPALM was performed using a custom-built setup as described previously (Lu et al., 2014). Imaging was conducted at $50 \mathrm{~Hz}$, with $4 \mathrm{~ms}$ laser pulses for 5000-10,000 frames. To account for lateral drift, a 3-plane $z$-stack ( $1.5 \mu \mathrm{m}$ thick) of PSDs marked by fluorescently tagged Homer1c was taken once before and after each single-molecule acquisition, a cross-correlation was calculated on maximum intensity projected images of the two sets, and experiments that yielded a lateral shift of more than one pixel $(100 \mathrm{~nm})$ were discarded from further analysis.

The single-molecule tracking method uPAINT was applied as reported by Giannone et al. (2010). FKBP- and FRB-containing proteins were cross-linked using $1 \mu \mathrm{M}$ rapalog (AP21967 or A/C Heterodimerizer, Clontech). Neurons were preincubated in full culture medium at $37^{\circ} \mathrm{C}$ for $1-2 \mathrm{~h}$ with rapalog, and subsequent imaging was performed at $25^{\circ} \mathrm{C}$ in extracellular imaging solution. ATTO647N-conjugated anti-GFP nanobodies (GFPBooster-647N, Chromotek) were bath applied to a final concentration of 300-500 pM once the first stretch of synapses was identified for each coverslip. BSA (A7030, Sigma-Aldrich) was bath applied to a 
A

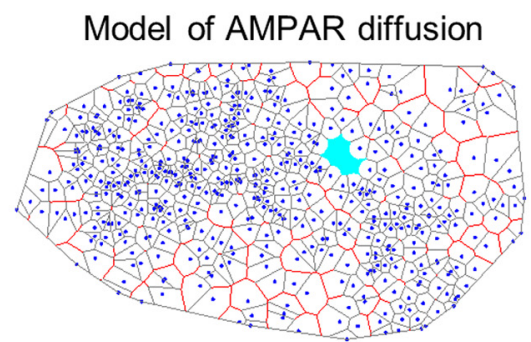

- PSD-95 molecule

- AMPAR diffusion

- Conducting path

- Blocked path

C

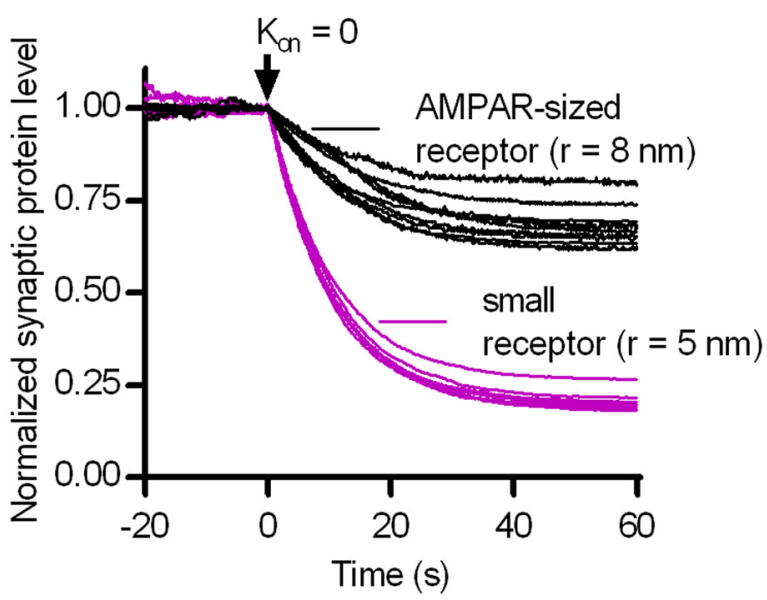

B

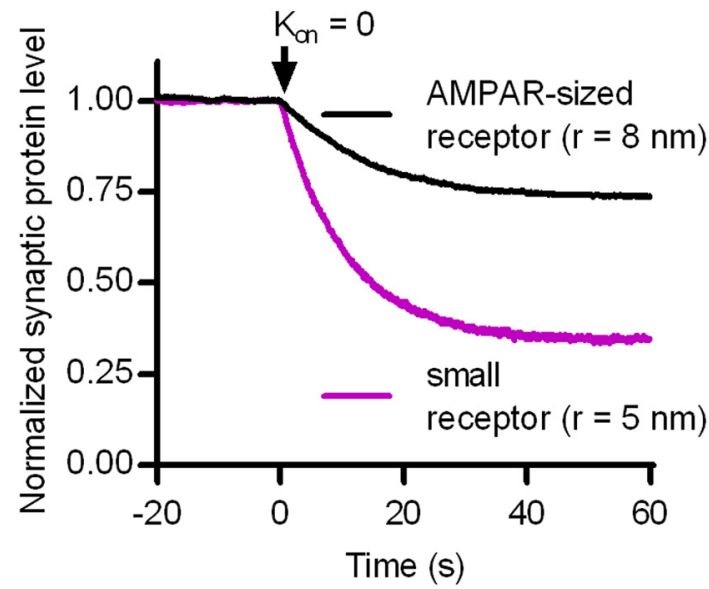

D

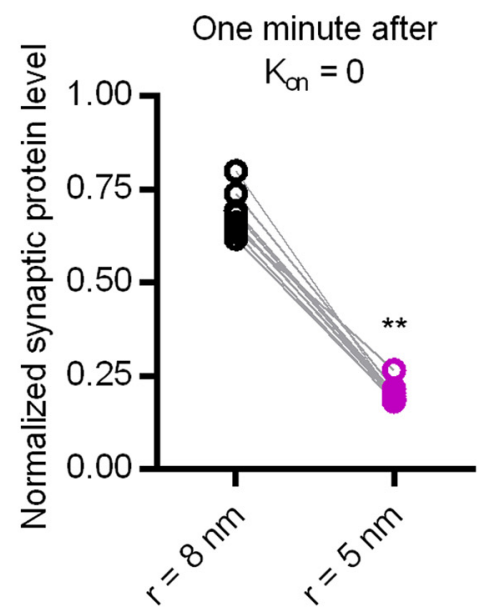

E

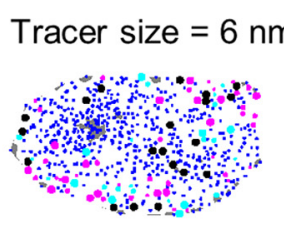

Tracer size $=13 \mathrm{~nm}$

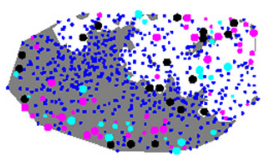

- PSD-95 - NMDAR

- Other immobile proteins

White region denotes conducting area
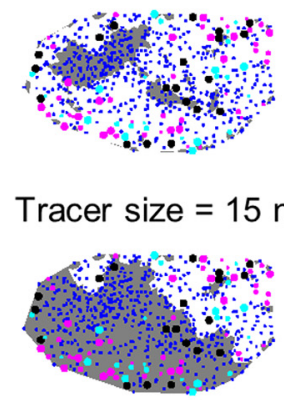

Tracer size $=10 \mathrm{~nm}$

Tracer size $=15 \mathrm{~nm}$

Adhesion molecules

$\mathbf{F}$

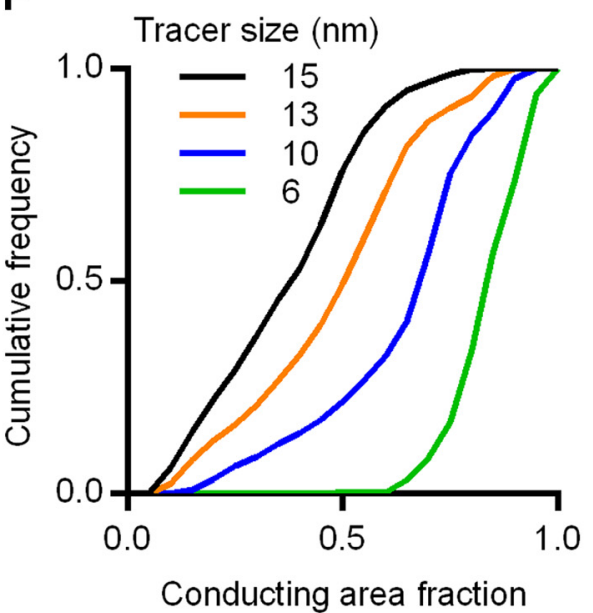

Figure 1. Synaptic crowding can retain membrane proteins in a size-dependent manner. $\boldsymbol{A}$, Single example of simulated AMPAR-sized tracer diffusion (cyan) in a measured map of PSD-95 molecules (black) (MacGillavry et al., 2013). Conducting (red line segments) and blocked paths (gray line segments) of diffusion are predicted through percolation theory and Voronoi tessellation of PSD-95-occupied regions. $\boldsymbol{B}$, Synaptic level of large (black) and small (magenta) receptor before and after acutely abolishing receptor-scaffolding protein binding $\left(\mathrm{K}_{\text {on }}=0\right)$ in a single measured PSD-95 map. C, Same simulation as $\boldsymbol{B}$ performed for multiple measured PSD-95 maps $(n=9)$. D , Paired statistical comparison of synaptic levels of large and small receptors 1 min after turning off binding in multiple measured PSD-95 maps ( $n=9$ synapses). ${ }^{* *} p=0.0039$ (Wilcoxon matched-pairs signed rank test). $\boldsymbol{E}$, Conducting area (white) estimated by Voronoi tessellation, predicting all possible paths a tracer of increasing size was allowed to take in an environment filled with PSD-95 molecules measured from PALM, along with other abundant proteins whose relative numbers were estimated from mass spectroscopy (Cheng et al., 2006). F, Cumulative likelihood of conducting area fraction of differently sized tracers in 100 measured PSD-95 maps (MacGillavry et al., 2013).

final concentration of $0.5 \%$ to ameliorate nonspecific interactions of nanbodies with untransfected cells and the coverslip surface. A twocolor single-molecule imaging setup that splits the emission of red and far-red bands was used as described by MacGillavry et al. (2013), in order to minimize mechanical vibration after acquiring the first and before acquiring the last $Z$-stack of PSDs. To image subsequent fields of the same cell or coverslip, the bound nanobodies were photobleached first. 
A

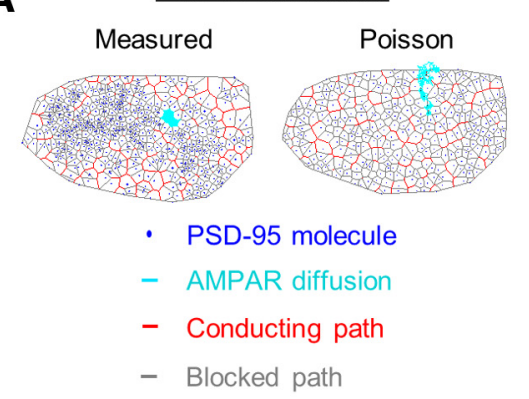

C

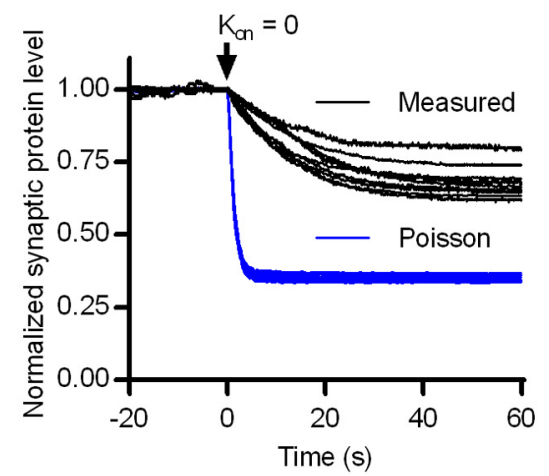

B

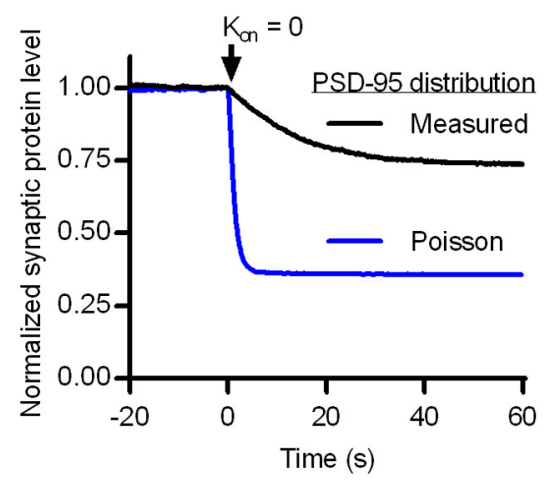

D

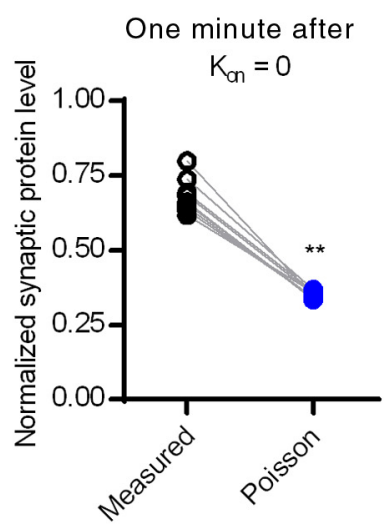

Figure 2. The arrangement of PSD-95 molecules can prolong the retention of synaptic AMPAR. A, Single examples of AMPARsized tracer diffusion in a measured distribution of PSD-95 molecules (left) and in randomly distributed PSD-95 molecules (right) (MacGillavry et al., 2013). B, Synaptic receptor level before and after acutely abolishing receptor-scaffolding protein binding $\left(K_{\text {on }}\right.$ $=0$ ) in a measured PSD-95 map (black) and its convex-hull matched Poisson distributed PSD-95 map (blue). C, Simulation as done in $\boldsymbol{B}$ repeated for multiple measured PSD-95 maps $(n=9)$ and their convex-hull-matched Poisson distributed PSD-95 maps $(n=$ 9). D, Paired statistical comparison of synaptic receptor levels 1 min after turning off binding in measured PSD-95 maps and their hull-matched Poisson PSD-95 maps ( $n=9$ synapses). ${ }^{* *} p=0.0039$ (Wilcoxon matched-pairs signed rank test).

Single-molecule localization, tracking analysis, and diffusion coefficient calculation. All data analysis was performed offline using custom routines in MATLAB (The MathWorks). Algorithms for determining molecule locations and criteria for filtering molecules to be considered in further analysis were applied as described previously (MacGillavry et al., 2013). Criteria for defining a track were described by Lu et al. (2014). Using a tracking radius of $500 \mathrm{~nm}$ permitted monitoring of molecules with diffusion coefficients up to $3 \mu \mathrm{m}^{2} / \mathrm{s}$, the upper bound for AMPARs as estimated by other groups (Bats et al., 2007; Hoze et al., 2012; Nair et al., 2013). Synaptic tracks were defined as tracks or periods of tracks that were within the border of the PSD, defined as in Lu et al. (2014). Nonsynaptic tracks were defined as tracks or periods of tracks that were within a neuronal border but outside enriched regions or clusters as defined by a Voronoi-based segmentation program, SR-Tesseler (Levet et al., 2015). Briefly, each imaging field was subdivided into a number of polygonal regions centered on the localizations detected in smtPALM or uPAINT. A density metric $\delta_{i}^{1}$ was computed for each localization, defined as the localization density within the total area composed of the polygon surrounding the localization and the immediate edge-sharing polygons. Localizations within the neuron were defined by thresholding the localizations with $\delta_{\mathrm{i}}^{1}>2 \delta_{\mathrm{I}}$, where $\delta_{\mathrm{I}}$ is the average localization density in the whole imaging field. Localizations within clusters were defined by the threshold $\delta_{\mathrm{i}}^{1}>2 \delta_{\mathrm{N}}$, where $\delta_{\mathrm{N}}$ is the average localization density of neuronal localizations. In general, some of the clustered localizations colocalized with the synaptic marker Homer1c, but some did not. Potential contributions include synapses with low Homer1C expression, regions with a high density of endocytic adaptor molecules, sites of plasma membrane-ER apposition, or zones of dense cortical cytoskeleton. Because the precise origins of the clustered nonsynaptic localizations were outside the scope of this study and difficult to distinguish, we discarded them from further analysis. Nonsynaptic tracks were formed from unclustered neuronal localizations.

For PALM, the effective diffusion coefficient $\left(D_{\text {eff }}\right)$ was calculated for tracks that persisted at least 4 frames on a weighted linear fit of the first four points of the mean square displacement (MSD) plots as described by Lu et al. (2014). For uPAINT in which tracks are typically longer and MSD plots are linear up to $8-10$ frames, D was calculated for tracks that persisted at least 8 frames. MSD curves of synaptically located molecules in uPAINT experiments were determined from whole tracks or periods of tracks that spent at least 30 contiguous frames within the PSD borders. Statistical comparisons were done on MSD $(t=30 \times 20 \mathrm{~ms})$ as a relative, not absolute, means of comparing the explored areas.

\section{Statistics}

Where means are presented, the accompanying errors are the SEM; additionally, these data were normally distributed according to the Shapiro-Wilk normality test. Where box-andwhisker plots are presented, the middle bar represents the median, the upper and lower limit of the boxes denote the interquartile range, and the whiskers extend to 5\% and 95\% of the distribution; additionally, these data were not normally distributed according to the Shapiro-Wilk normality test. Different sets of statistical tests were used for normally and non-normally distributed data. Pairwise statistical tests were performed using unpaired $t$ test with Welch's correction for normally distributed data; they were performed using MannWhitney $U$ test for non-normally distributed data. For experiments with more than two conditions, one-way ANOVA was used to test for overall differences among means of normally distributed data, whereas Kruskal-Wallis one-way ANVOA was used to test for overall differences among medians of non-normally distributed data. For partial and full synapse FRAP experiments, two-way ANOVA was used. For all omnibus tests, a Bonferroni correction was used for post hoc pairwise comparisons. Kolmogorov-Smirnov tests were applied for cumulative frequency distributions. In all cases, means (or medians) were considered significantly different if the test reported $p<0.05$.

\section{Results}

Transmembrane protein size influences its access to areas within the PSD

Emerging data demonstrate that PSD-95, the most abundant of synaptic scaffolding proteins, can be arranged in multiple welldefined, 80-nm-wide clusters within the excitatory synapse (MacGillavry et al., 2013; Nair et al., 2013). To predict how this type of arrangement can influence the mobility and retention of critically important transmembrane proteins, we first developed a minimalist model of the PSD, which incorporated coordinates of PSD-95 molecules (Fig. 1A, blue dots) measured by PALM (MacGillavry et al., 2013). In addition to PSD-95 molecules, the model PSD map contained a random placement of other abundant synaptic proteins (i.e., NMDARs, adhesion molecules, and other relatively immobile proteins such as potassium channels), the relative concentrations of which were based on massspectroscopy estimates (Cheng et al., 2006). We modeled the AMPARs to be hourglass shaped, its external, transmembrane, 
A

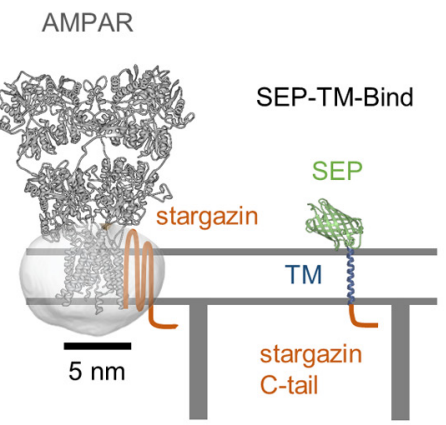

PSD-95
B

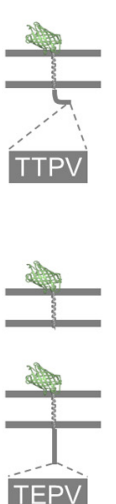

C

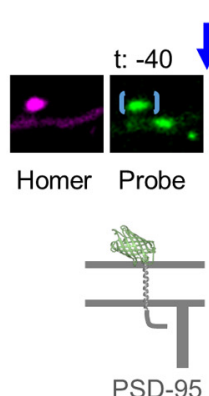

PSD-95 $\frac{1}{0}$ 10 $135 \mathrm{~s}$
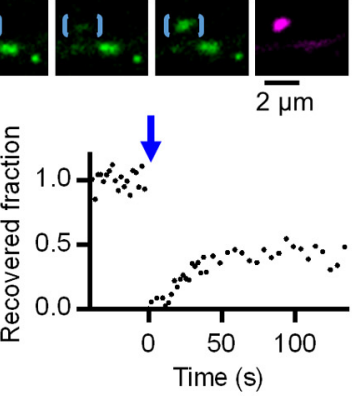

E

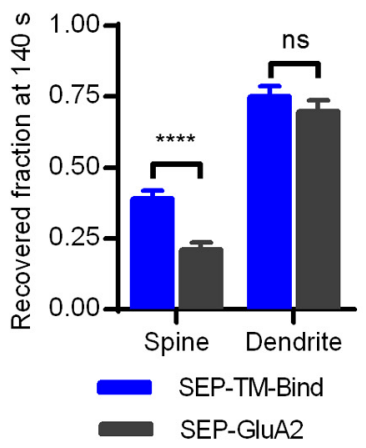

F

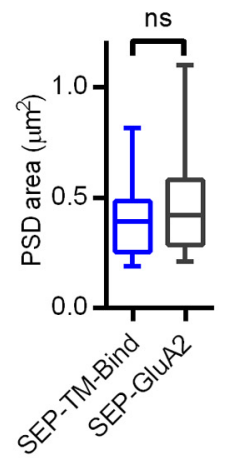

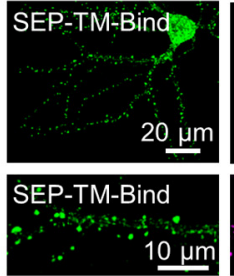
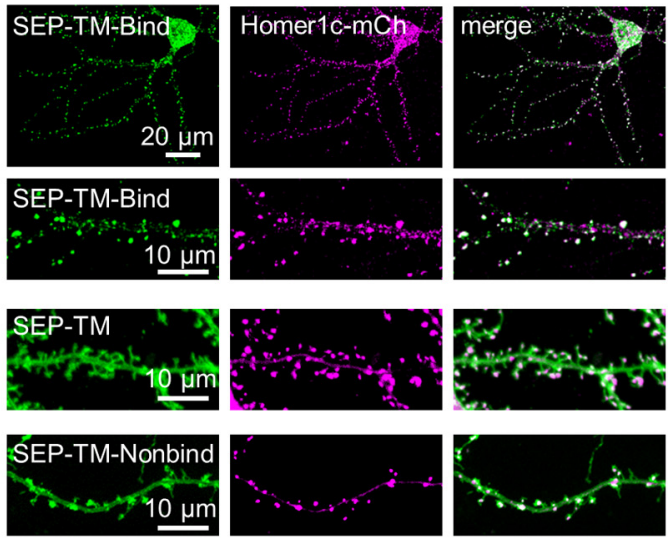

D

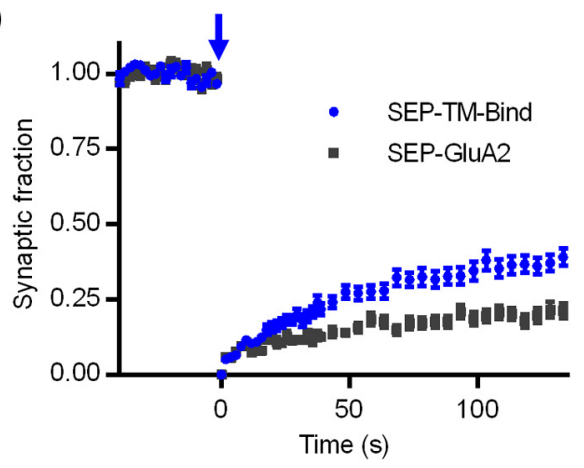

G

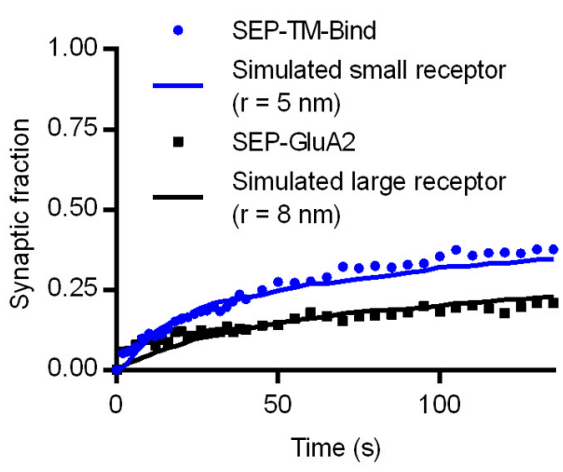

J

H

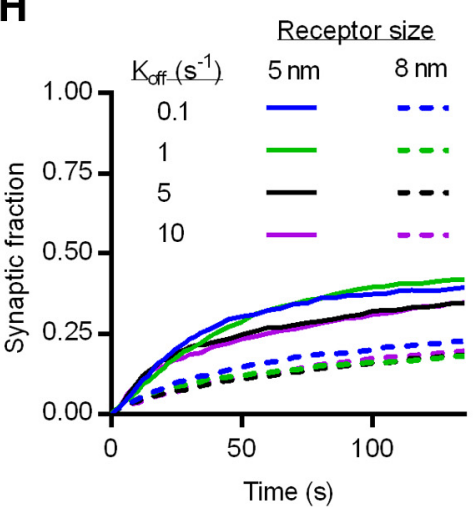
Receptor size (nm)

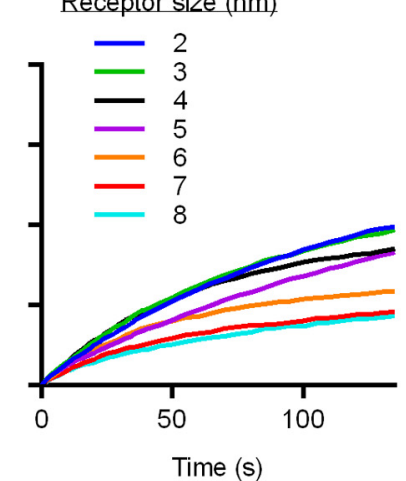

Figure 3. Small- and large-membrane proteins exchange differently on the surface membrane of dendritic spines. $A$, Schematic of AMPAR crystal structure and minimalist membrane protein (SEP-TM-Bind) drawn to scale. SEP-TM-Bind is a fusion protein of three parts: SEP, a pH-sensitive GFP protein; TM, the transmembrane domain of PDGFR; and the binding component, which is the cytoplasmic tail of Stargazin. $\boldsymbol{B}$, The SEP-TM-Bind was trafficked to the synapse (top and second row) in dissociated hippocampal cultured neurons; the SEP-TM, which lacks any intracellular domain, was expressed with uniform distribution across dendritic surface (second to last row); and the SEP-TM-Nonbind (in which Thr at the -2 position was mutated to be Glu) was trafficked with uniform surface distribution as well (last row). $\boldsymbol{C}$, An example of FRAP to assess the mobility of SEP-TM-Bind on spines. $\boldsymbol{D}$, Averaged recovery curves \pm SEM for small- and large-membrane proteins in spines ( $n=42 / 11 / 2$ ROIs/neurons/cultures for SEP-TM-Bind, 37/10/2 for SEP-GluA2). $E$, Recovered fractions at 140 s in spines $\left({ }^{* * * *} p<0.0001\right.$, unpaired $t$ test, $n=42 / 11 / 2$ for SEP-TM-Bind, 37/10/2 for SEP-GluA2) and dendritic segment (ns, Not significant, $p=0.31, n=27 / 9 / 2$ for SEP-TM-Bind, 33/10/2 for SEP-GluA2). $\boldsymbol{F}$, Areas determined (Figure legend continues.) 
and internal radii consistent with sizes measured using EM tomography (Chen et al., 2008) and single-particle EM (Nakagawa et al., 2005). Details on the shapes and sizes of AMPARs, PSD-95, and other components of the PSD model can be found in Materials and Methods. We calculated the possible diffusion trajectories of AMPARs within the PSD by using two independent modeling methods: Monte Carlo simulation of Brownian dynamics and geometric modeling of potential diffusion paths using percolation theory (for programming details, see Materials and Methods). Geometric modeling showed that a significant fraction of the PSD map was excluded from receptor passage (Fig. 1A, gray lines). Monte Carlo simulation of receptor diffusion showed that a single AMPAR initiated within the PSD can sometimes be trapped (Fig. 1A, cyan region). Regions of trapped diffusion overlapped with regions that lacked percolation paths, even in the absence of binding. This initial observation predicted that the PSD could trap receptors, even in the absence of receptor-scaffold interaction.

To test this prediction, we allowed receptor concentration to reach steady state through receptor-scaffolding binding, and then turned off the binding interaction $\left(\mathrm{K}_{\mathrm{on}}=0\right)$. In the PSD map shown in Figure $1 A$, we found that majority $(\sim 75 \%)$ of AMPARs remained in the PSD, 1 min after abolishing receptorscaffold binding (Fig. 1B, black curve), consistent with the small reduction in MEPSC amplitude measured in the presence of a peptide that disrupted the interaction between AMPAR and PSD-95 (Sainlos et al., 2011). To investigate whether the retention of AMPARs can be influenced by the protein bulk alone, we repeated the simulation using a "small receptor," with an intracellular domain radius of $5 \mathrm{~nm}$ as opposed to $8 \mathrm{~nm}$ in the full AMPAR complex. We found that a much smaller fraction of small receptors remained in the synapse than AMPARs did in the absence of binding (Fig. 1B, magenta curve). This effect was robust in a randomly selected subset of PSD maps ( $n=9$ maps; mean \pm SEM of small receptor $20 \pm 0.9 \%$; large receptor $68 \pm$ $1.9 \% ; p=0.0039$; Fig. $1 C, D)$. In a model of cylindrical tracer diffusion on 2D membrane, Saffman and Delbrück (1975) predicted that lateral diffusion coefficient is directly proportional to $\log (1 / R)$, where $R$ is the radius of the cylindrical tracer. This relation suggests a weak dependence of diffusion coefficient on particle size, which was experimentally shown by Kucik et al. (1999). The difference of synaptic retention between small and large tracers observed here was larger than what could be explained by obstacle-independent change in diffusion on 2D membrane, suggesting that PSD-95 crowding can regulate the mobility and retention of AMPARs and other critically important transmembrane proteins based on the sizes of their protein bulks.

To investigate whether macromolecular crowding can influence the positioning of receptors based on protein size, we estimated all possible moving paths of differently sized cylindrical tracers in measured PSD-95 maps based on Voronoi methods used to study percolation (Saxton, 2010). We found that the smaller the tracer, the larger the synaptic region it was able to

$\leftarrow$

(Figure legend continued.) from confocal images of Homer1c-mCherry coexpressed with either SEP-TM-Bind or SEP-GluA2 in FRAPed spines ( $p=0.18$, Mann-Whitney test, $n=42 / 11 / 2$ for SEP-TM-Bind, 37/10/2 for SEP-GluA2). G, Simulated FRAP to fit parameters of $K_{\text {on }}$ and $K_{\text {off }}$ in a measured PSD-95 map. $\boldsymbol{H}$, Changing binding dissociation rate $\left(\mathrm{K}_{\text {off }}\right)$ by 2 orders of magnitude did not remarkably alter the simulated FRAP spines containing either small $(r=5 \mathrm{~nm})$ or large tracer $(r=8 \mathrm{~nm}) . I$, Changing $\mathrm{K}_{\text {flip }}$ by 2 orders did not significantly alter spine FRAP.J, Changing the receptor size within one order of magnitude significantly altered spine FRAP. ${ }^{* * *} p<$ 0.0001. ns, Not significant. traverse (Fig. 1E,F), which suggests that the distribution of receptors within the synapse can be regulated in a manner dependent on the sizes of the diffusing proteins.

We next considered whether the conservative retention of AMPARs is specific to the heterogeneous arrangement of PSD-95 molecules. To test this in the model, we allowed the receptor concentration to reach steady state either in a map of measured PSD-95 locations or in a boundary-matched map of randomly distributed PSD-95 locations (Fig. 2A), and then we measured receptor concentrations after turning off receptor-scaffold binding (Fig. 2B). Interestingly, receptors escaped quicker and to a larger extent from PSDs with randomized distribution of PSD-95 molecules than from PSDs with measured distribution of PSD-95 molecules (Fig. 2C,D). This result suggests that PSD can tune the density of membrane proteins by modulating the arrangement of PSD-95 molecules.

\section{Transmembrane protein mobility depends on protein size in living synapses}

To examine systematically the influence of protein size on protein mobility in synapses of live neurons, we first created a singlepass transmembrane probe molecule containing a SEP on the extracellular domain and the cytoplasmic tail of Stargazin (SEPTM-Bind) (Fig. 3A). Akin to the AMPAR, this minimal probe was prominently trafficked to synapses marked by cotransfected Homerlc tagged with mCherry (Fig. 3B). Synaptic accumulation required the Stargazin C-tail, as a second probe lacking this domain (SEP-TM) trafficked with a nearly uniform distribution across the neuronal surface (Fig. 3B). More specifically, synaptic accumulation required the PDZ ligand on the Stargazin C-tail, as a third probe (SEP-TM-Nonbind) containing a single point mutation on threonine at position -2 (TTPV to TEPV) trafficked with a uniform distribution across the cell surface as well (Fig. 3B, bottom), consistent with previous reports, which demonstrated that the same mutation abolished the binding between Stargazin and PSD-95 (Chetkovich et al., 2002; Choi et al., 2002). To test the model prediction that transmembrane protein mobility within the synapse depends on protein size, we first measured fluorescence recovery of the small-transmembrane probe and AMPAR after photobleaching single spines (Fig. 3C,D). SEPGluA2 recovered on average 21\% after $140 \mathrm{~s}$, similar to previous reports (Ashby et al., 2006; Kerr and Blanpied, 2012); however, SEP-TM-Bind recovered nearly twice this amount (Fig. 3E). Both proteins recovered similar amounts in the dendritic shaft (Fig. $3 E$ ). As it could be the case that smaller synapses correlate with faster recovery, we examined the PSD areas within the bleached spines by measuring the width of the Homer1c-mCherry image. The PSD areas were not different (Fig. $3 F$ ), the recovery of SEPTM-Bind at $140 \mathrm{~s}$ after bleaching was weakly negatively correlated with PSD area $\left(r^{2}=0.17, p=0.0064, n=42\right)$, and the recovery of SEP-GluA2 at $140 \mathrm{~s}$ after bleaching was not correlated with PSD area $\left(r^{2} \ll 0.001, p=0.97, n=37\right)$, suggesting that a difference in synapse size cannot account for the recovery difference in spines between the two groups.

It is possible that the difference of recovered fractions in spines between SEP-GluA2 and SEP-TM-Bind could be explained by a potential difference in affinity for PSD-95. To examine this notion formally, we turned to computer modeling (and see also Fig. 9). The kinetic rates of the model (i.e., $K_{\text {off }}$, receptorscaffold binding affinity; and $\mathrm{K}_{\text {flip }}$, the likelihood to reset photobleached receptors back to fluorescence when they hit the boundary of simulated spine head area) were initialized by minimizing the difference between the simulated and the measured 
recovery curves (Fig. $3 G$ ). Varying $\mathrm{K}_{\text {off }}$ by 2 orders of magnitude had negligible influence on the time course and steady state of simulated recoveries, but changing the tracer size by a factor of 2 yielded a consistent difference in recovery for a large range of $\mathrm{K}_{\text {off }}$ values (Fig. $3 H$ ). This suggests that the size of the receptor protein is more likely than the receptor-scaffold binding affinity to influence receptor mobility. Altering $\mathrm{K}_{\text {flip }}$ by 2 orders of magnitude yielded notable change in the recovery speed of the small tracer but had negligible influence on the steady state of the recovery for both the large and the small tracer (Fig. 3I). $\mathrm{K}_{\text {flip }}$ crudely accounts for factors, such as spine geometry and synaptic-extrasynaptic protein concentration gradient; thus, the result suggests that these factors are unlikely to explain the difference in the mobile fraction after 2 min of exchange. Importantly, tracers that were no more than 4 times smaller than the AMPAR recovered more than twice as much after $2 \mathrm{~min}$ (Fig. 3J), suggesting that membrane protein size can be a major determinant of mobility.

In further experiments, we found that this difference persisted at longer periods following photobleaching (Fig. $4 A, B$ ). The small binding probe recovered more fully than AMPARs both transiently (2 min after bleaching) and near steady state (52 min after bleaching) (Fig. 4C), suggesting that, compared with the larger AMPARs, the small probe diffuses faster and has a smaller proportion stabilized in the spine.

To address whether the spine-todendrite concentration gradient or an altered synapse size induced by probe expression might contribute to these observed differences in mobility, we measured the PSD area and spine enrichment (defined in Materials and Methods) in the same spines that were followed for FRAP. The PSD areas were not different between spines expressing SEP-TM-Bind and SEPGluA2 (Fig. 4D). The recovered fraction of the small probes 2 min after bleaching was weakly inversely proportional to PSD area $\left(r^{2}=0.26\right)$, and that of AMPARs was not correlated with PSD area (Fig. 4E). Fifty-two minutes after bleaching, the recovered fractions of both proteins were not correlated with PSD areas (Fig. $4 F$ ), indicating that the fraction of molecules exchanging in and out of the spine was unaffected by synapse size. The spine enrichment was not different between spines expressing the small probes and AMPARs (Fig. 4G). The transient and steady-state
A

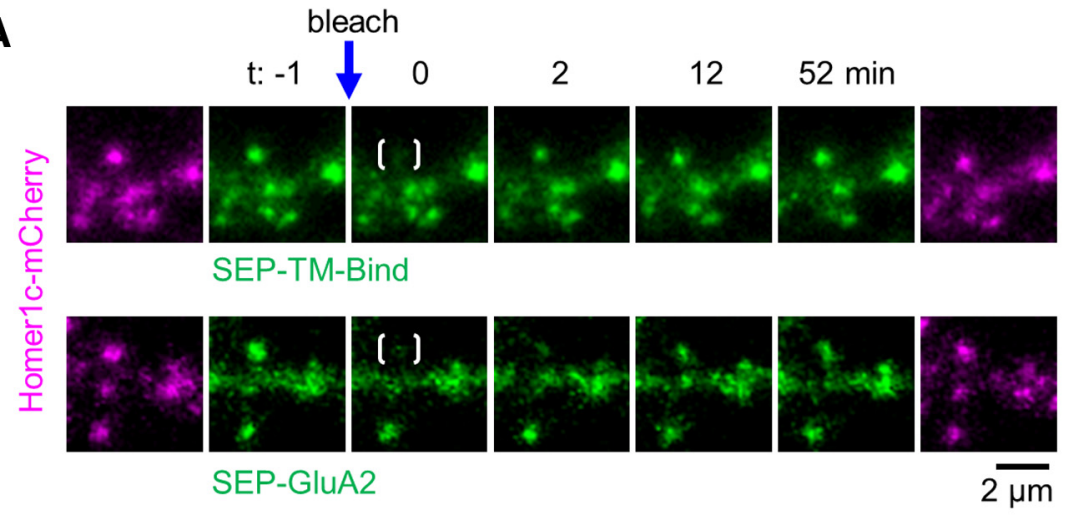

B

C
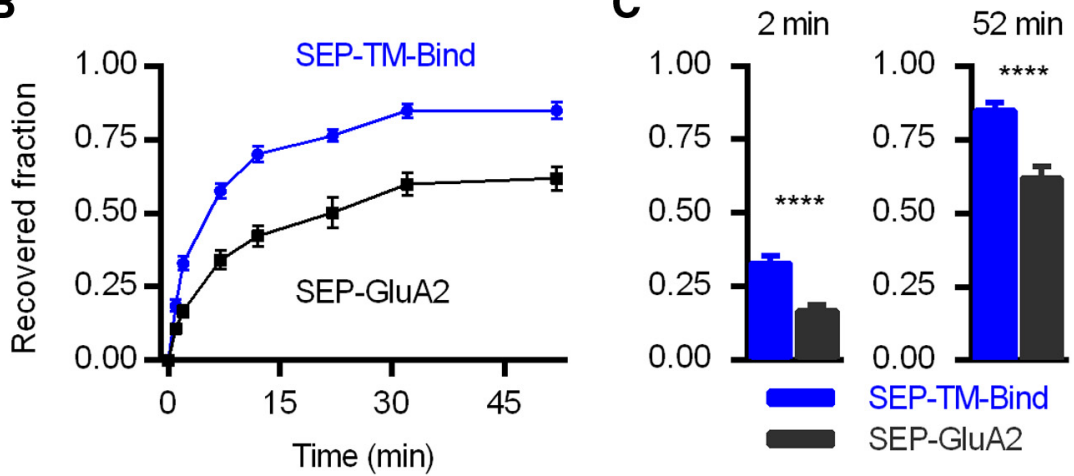

D

E

$2 \min$
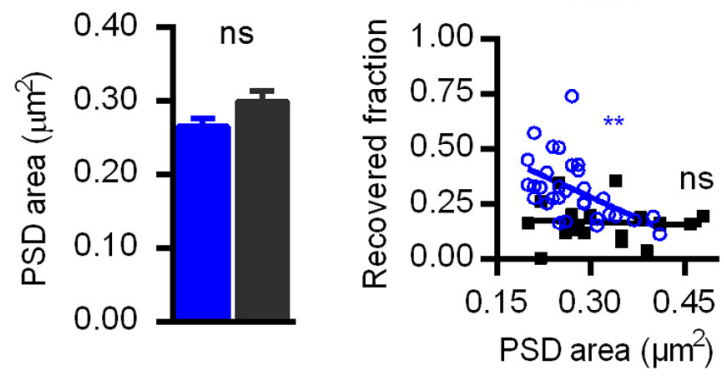

F

$52 \mathrm{~min}$
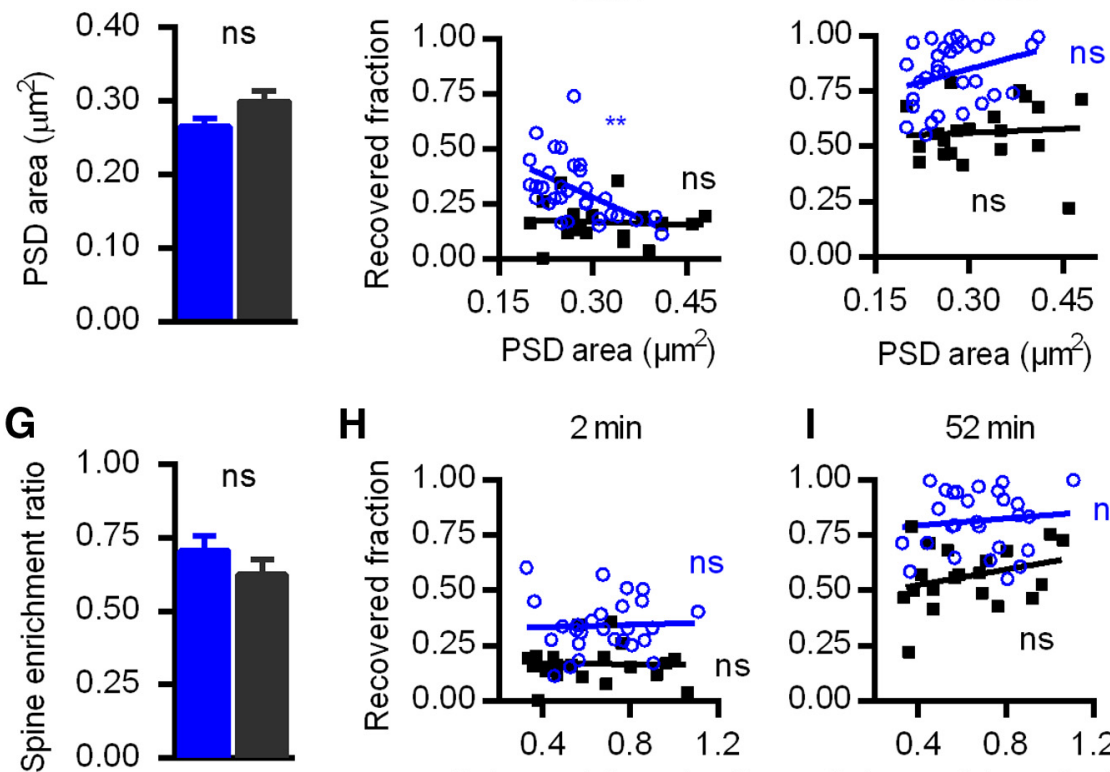

H

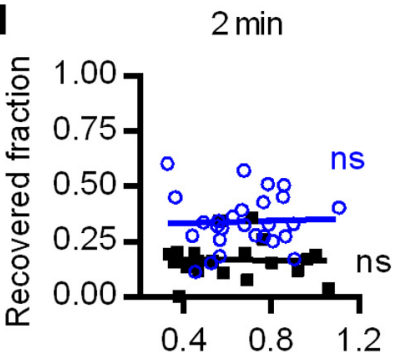

Spine enrichment ratio

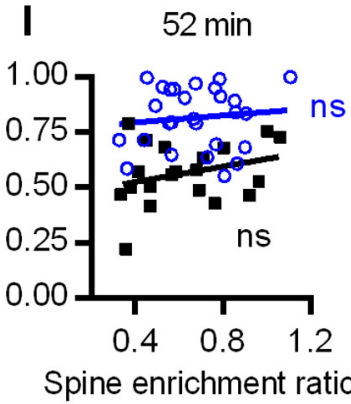

Figure 4. The surface mobility of AMPAR in spines is unrelated to synapse size or spine-dendritic concentration gradient; that of the small probe is weakly related to synapse size. $A$, Representative FRAP images of DIV20-DIV21 hippocampal neurons cotransfected with Homer1 C-mCherry, and either SEP-TM-Bind or SEP-GluA2. Bracketed spines were photobleached. $\boldsymbol{B}$, Recovery curves of bleached SEP-TM-Bind ( $n=35$ spines $/ 5$ neurons) and SEP-GluA2 $(n=20 / 3)$ from 3 separate cultures. C, Recovered SEP fractions of SEP-TM-Bind and SEP-GluA2 in spines after 2 and $52 \mathrm{~min}(p<0.0001)$. D, PSD areas of bleached SEP-TM-Bind and SEP-GluA2 $(p=0.0732) . E$, PSD area and transient $(2 \mathrm{~min})$ recovery level of each bleached SEP-TM-Bind (circles, $r^{2}=0.26,{ }^{* *} p=0.0038$ ) and SEP-GluA2 (squares, $\left.r^{2}=0.0051, p=0.77\right) . F$, The PSD area and near-steady-state $(52 \mathrm{~min}$ ) recovery level of bleached SEP-TM-Bind $\left(r^{2}=0.091, p=0.10\right)$ and SEP-GluA2 $\left(r^{2}=0.090, p=0.77\right) . G$, Spine enrichment of bleached SEP-TM-Bind and $\operatorname{SEP}-\operatorname{GluA2}(p=0.255)$. $\boldsymbol{H}$, The spine enrichment and transient $(2 \mathrm{~min})$ recovery of bleached SEP-TM-Bind $\left(r^{2}=0.0018, p=0.83\right)$ and SEP-GluA2 $\left(r^{2}<0.001, p=0.91\right) . I$, The spine enrichment and near-steady-state $(52 \mathrm{~min})$ recovery of bleached SEP-TM-Bind $\left(r^{2}=0.011, p=0.60\right)$ and SEP-GluA2 $\left(r^{2}=0.086, p=0.21\right) . \boldsymbol{D}-\mathbf{I}$, Color coding is the same as in $\boldsymbol{C} . \boldsymbol{B}-\boldsymbol{D}, \mathbf{G}$, Data are mean \pm SEM; statistical test was unpaired $t$ test. ${ }^{* * *} p<0.0001$. ns, Not significant. 
recovery levels were not correlated with the spine enrichment in spines expressing either protein (Fig. 4 H,I). Together, both simulated and experimental FRAP results support the notion that receptor size is a prominent factor in limiting receptor mobility in the dendritic spine.

\section{Small- and large-membrane proteins traverse the PSD at different rates in live neurons}

Spine geometry will influence recovery after photobleaching the entire spine and synapse together (Ashby et al., 2006; Simon et al., 2014). To assess mobility within synapses, we first used photobleaching by targeting just part of single synapses and monitoring recovery within this subregion of the PSD (Kerr and Blanpied, 2012) (Fig. 5A). Fully bleached synapses will recover fluorescence via exchange with the extrasynaptic unbleached population. However, in the targeted region of partially bleached synapses, recovery will proceed more quickly than this only if mobility of the probe within the synapse (subsynaptic mobility) is substantial enough to permit the synaptic unbleached pool to distribute to the bleached region.

To formalize our expectations on the subsynaptic mobility of large- and small-membrane proteins, we first modeled this experiment. To simulate membrane protein dynamics, we modeled tracer diffusion by Monte Carlo simulations of Brownian dynamics (see Materials and Methods) within a measured PSD-95 distribution and in surrounding extrasynaptic space. In the case of full synaptic bleaching, all particles within the convex hull of the measured PSD-95 molecules (synaptic region) were set to be nonfluorescent, whereas ones outside remained marked as fluorescent. The number of fluorescent particles within the synaptic region were recorded as a function of time to calculate the full synapse FRAP curve (Fig. 5B, solid lines). In the case of partial synaptic bleach, particles within half of the synaptic region were considered bleached. The number of fluorescent particles within the bleached half were recorded over time to calculate the half synapse FRAP curve (Fig. 5B, dotted lines). The recovery dynamics of AMPAR-sized tracers $(r=8 \mathrm{~nm})$ were nearly identical after half-synapse bleaching and after full synapse bleaching (17\%$18 \%$ after $40 \mathrm{~s}$; Fig. $5 \mathrm{~B}$, black lines). On the other hand, the smaller tracers $(r=5 \mathrm{~nm})$ recovered more after half-synapse bleaching than after full synapse bleaching $(45 \%$ and $39 \%$ at $40 \mathrm{~s}$ after half and full synapse bleach, respectively; Fig. 5B, blue curves).

This result is useful to consider because recovery after photobleaching in synapses depends on several factors. The rate of recovery will depend on the extrasynaptic probe enrichment as well as extrasynaptic mobility. In addition, an important parameter is how quickly synaptic receptors explore the PSD to find an exit route compared with how fast exited receptors get replaced. In this simulation, several key variables that could influence the recovery were kept identical for both tracers: the PSD-95 distribution, the binding affinities, the true diffusion coefficient (D) of the molecule in the synapse, and the total number of tracers. In addition, the enrichment ratio of both tracers at the equilibrated state before photobleaching reached the same value of $\sim 4: 1$ (inside:outside the PSD). The only difference was the diameters of the tracers, which thus could access different fractional areas within the synapse. Therefore, the simulated subsynaptic bleaching results indicate that the conduction area or routes of passage within the synapse can contribute significantly to intrasynaptic recovery.

To test this in living synapses, we performed partial synaptic photobleaching in neurons expressing either SEP-GluA2 or SEP-
TM-Bind (Fig. 5C). Following full synapse photobleaching, we detected little fluorescence recovery after 5 s (3.2 $\pm 0.7 \%$ SEPGluA2, $2.3 \pm 0.4 \%$ SEP-TM-Bind; $n=15$ synapses in 7 neurons SEP-GluA2, 27 synapses in 9 neurons SEP-TM-Bind; Fig. 5D), suggesting that the rapidly exchanging populations of both largeand small-membrane proteins were very small in these synapses. After 11 min, $35.6 \pm 4.6 \%$ of synaptic AMPAR fluorescence was recovered, consistent with previous reports (Ashby et al., 2006; Sharma et al., 2006; Frischknecht et al., 2009; Arendt et al., 2010; Kerr and Blanpied, 2012), whereas $58.7 \pm 3.8 \%$ of synaptic membrane probe was recovered. These relative recovery fractions were as expected based on full-spine photobleaching (Fig. 2).

Following partial synapse photobleaching (performed during the same time series on neighboring synapses), recovery of AMPAR within the bleached subregion of the synapse was slightly higher after $5 \mathrm{~s}$ but not different after $11 \mathrm{~min}$ compared with fully bleached synapses $(6.7 \pm 1.6 \%$ and $34.0 \pm 6.2 \%$ for partial; $n=13$ synapses in 6 neurons; $p=0.037$ and $p=0.98$ compared with fully bleached synapses; Fig. $5 E$ ). However, recovery of the small-probe SEP-TM-Bind was higher after $5 \mathrm{~s}$ than in fully bleached synapses $(6.5 \pm 1.1 \%$ for partial; $n=18$ synapses in 9 neurons; $p=0.0012$ compared with fully bleached synapses; Fig. 5E, left). Importantly, it remained higher after $11 \mathrm{~min}$ (77.5 $\pm 1.7 \%$ for partial; $p=0.031$ compared with fully bleached synapses; Fig. $5 E$, right).

Image acquisition itself caused minimal photobleaching, as fluorescence intensity in neighboring unbleached synapses remained nearly constant $(94.5 \pm 4.3 \%$ of baseline for SEP-GluA2, $99.2 \pm 1.8 \%$ for SEP-TM-Bind after $11 \mathrm{~min}$ ). We assessed the bleached fraction in the synaptic subregion not targeted for photobleaching. It was determined first by outlining the entire synapse excluding the half-targeted for bleaching and then calculating the fluorescence lost immediately after photobleaching. We calculated this because the confocal laser spot inevitably affects part of the synapse unintended for bleaching, and we reasoned that the fraction of unbleached proteins could influence the recovery within the bleached region. However, we found that the bleached fractions in regions unintended for photobleaching were not different for both proteins (mean \pm SEM: $40 \pm 4 \%$ for SEP-GluA2, $36 \pm 4 \%$ for SEP-TM-Bind; unpaired $t$ test, $p=$ $0.17)$. Bleached fractions in the synaptic subregion targeted for photobleaching were also not different (mean \pm SEM: $67 \pm$ $2 \%$ for SEP-GluA2, $67 \pm 2 \%$ for SEP-TM-Bind; unpaired $t$ test, $p=0.88$ ). It is possible that this modest difference in bleached fractions between two subregions of the synapse resulted in a dynamic range that was insensitive to a significant subpopulation of AMPARs that exchanged laterally on the order of minutes within the synapse. Nonetheless, the difference in recovery between the two proteins was robust on a minute timescale (Fig. $5 D, E$ ).

It is possible that AMPARs were overall less mobile within the larger-than-average PSDs required to perform partial synaptic FRAP reliably. However, the synaptic areas were not different for the two proteins (Homer1c-mCherry area median [interquartile range] $\mu \mathrm{m}^{2}: 0.18$ [0.14-0.31] SEP-GluA2, 0.21 [0.14-0.28] SEP-TM-Bind, Mann-Whitney $U$ test $p=0.63$ ). Furthermore, the recovery fraction of SEP-GluA2 was uncorrelated with PSD area in both partial and full synapse bleaching schemes $\left(r^{2}=\right.$ $0.19, p=0.14, n=13$ PSDs for partial; $r^{2}=0.005, p=0.81, n=$ 15 full; Fig. 5F, left). Moreover, the recovery fraction of the small binding probe was uncorrelated with PSD area in the partial synapse bleaching case, and negatively but weakly correlated with PSD area in the full synapse bleaching case $\left(r^{2}=0.061, p=0.33\right.$, 
A

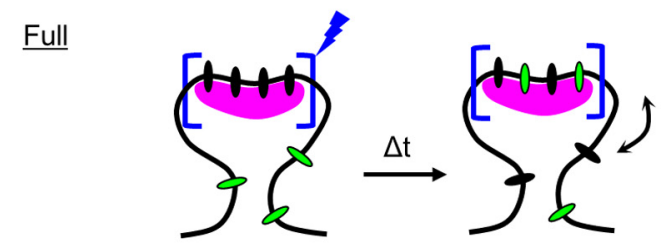

$\underline{\text { Partial }}$

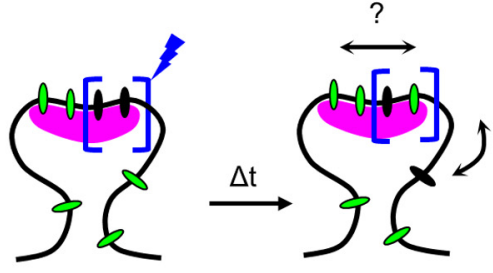

C

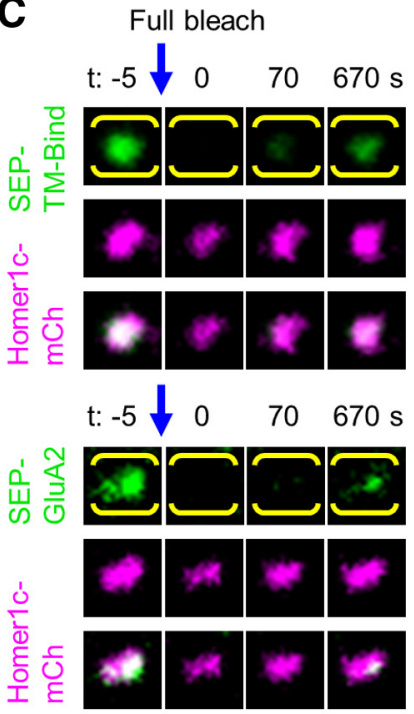

E

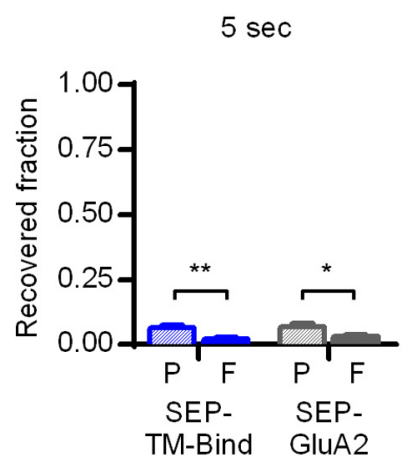

Partial bleach

t: -5 \ $0 \quad 70670 \mathrm{~s}$
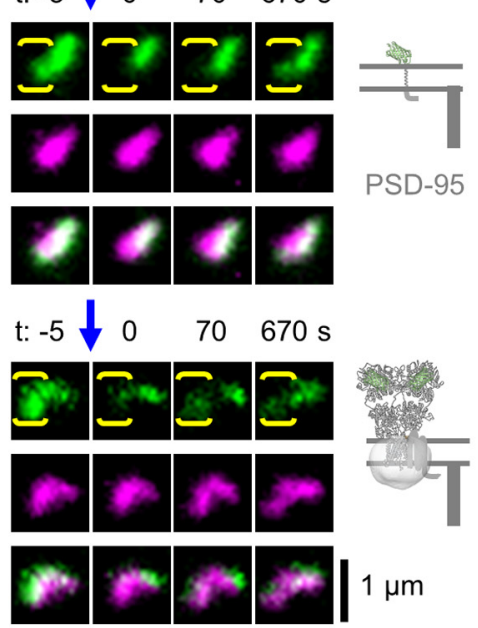

F

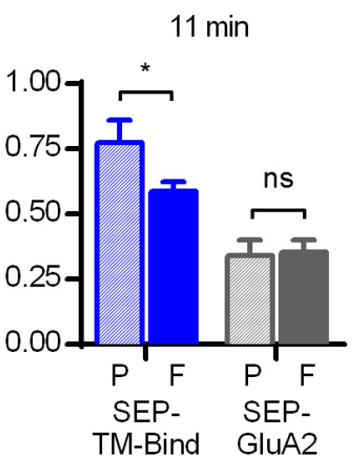

B

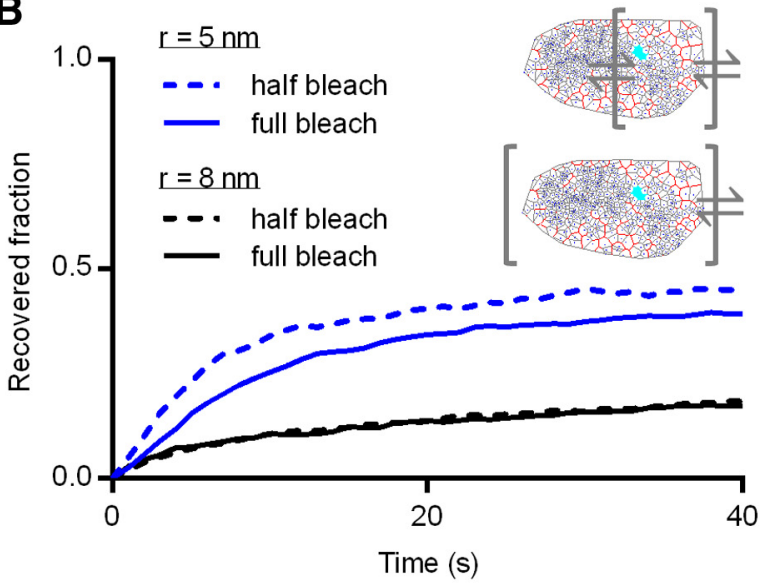

D

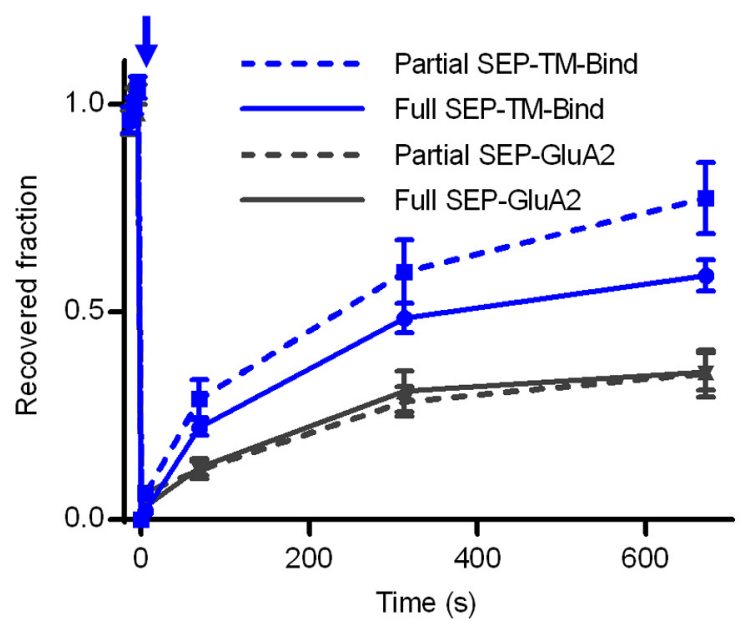

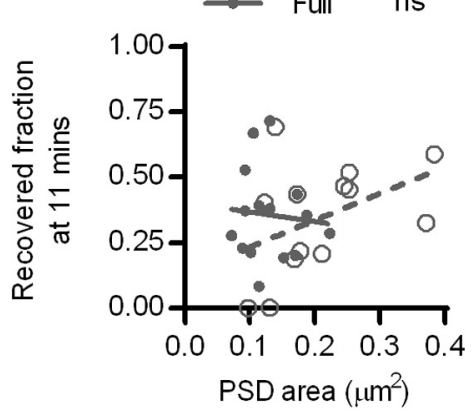

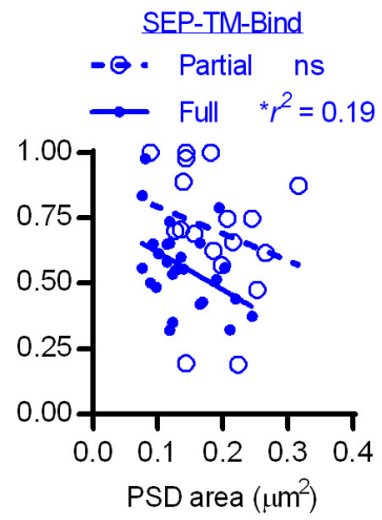

Figure 5. Small-membrane probe has higher subsynaptic mobility than AMPAR. $\boldsymbol{A}$, Partial synaptic FRAP can assess mobility of membrane proteins within the confine of a synapse. $\boldsymbol{B}$, Simulated partial synaptic and full FRAP for small $(r=5 \mathrm{~nm})$ and large tracer $(r=8 \mathrm{~nm})$. C, Example synapses expressing SEP-TM-Bind or SEP-GluA2 and Homer1c-mCherry where all or part of the synaptic SEP was photobleached. D, Top, SEP-TM-Bind or SEP-GluA2 fluorescence recovery in synapses targeted for full or partial synapse photobleaching. Data are mean \pm SEM. Experiments were interleaved in neurons coexpressing either SEP-TM-Bind or SEP-GluA2.E, SEP-TM-Bind or SEP-GluA2 fluorescence recovery at $5 \mathrm{~s}$ and 11 min after photobleaching. $\mathrm{n}_{\text {Bind, full }}=27$ PSDs $/ 9$ neurons $/ 2$ cultures, $\mathrm{n}_{\text {Bind, partial }}=18 / 9 / 2, \mathrm{n}_{\mathrm{GluA2} \text {,full }}=15 / 7 / 2, \mathrm{n}_{\mathrm{GluA2} \text {, partial }}=13 / 6 / 2 .{ }^{* *} p=0.0012,5 \mathrm{~s}$ (two-way ANOVA with Bonferroni's multiple comparisons test). ${ }^{*} p=0.037,5 \mathrm{~s}$ (two-way ANOVA with Bonferroni's multiple comparisons test). ${ }^{*} p=0.032$. ns, Not significant ( $\left.p>0.999\right)$ for 11 min. $\boldsymbol{F}$, Left, The PSD area and recovered fraction 11 min after partially or fully bleaching SEP-GluA2 in each synapse (not significant $p=0.14$ for partial, $p=0.81$ full). Right, After partially or fully bleaching SEP-TM-Bind in each synapse (not significant $p=0.33$ for partial, ${ }^{*} p=0.026$ full).

$n=18$ PSDs for partial; $r^{2}=0.19, p=0.026, n=27$ full; Fig. $5 F$, right). Thus, the difference in recovery between the two proteins as measured by partial synapse photobleaching was likely not caused by the selective use of large synapses.
In the partial bleaching assay, this difference in recovery between probes of two sizes could arise from a difference in the ratio of their diffusion coefficients in versus out of the synapse, or from their differential interactions with the PSD interior milieu that 
A

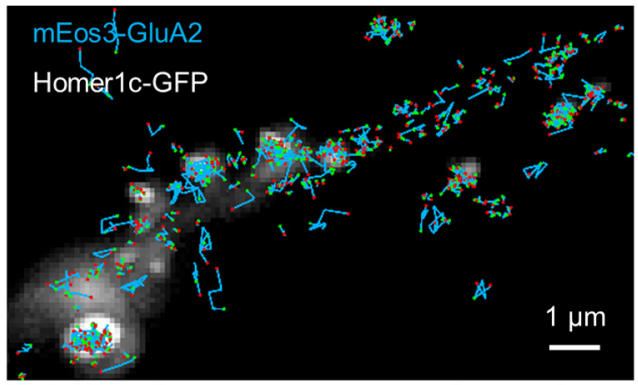

C

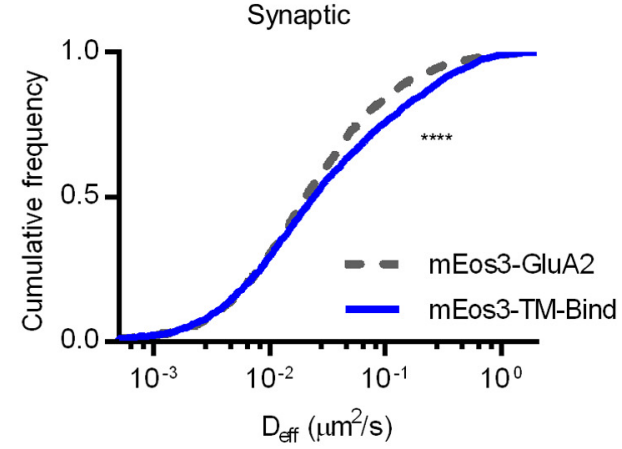

B

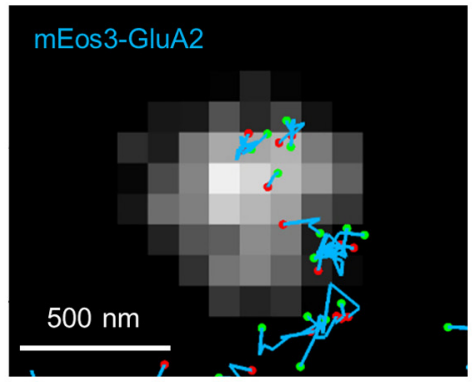

D

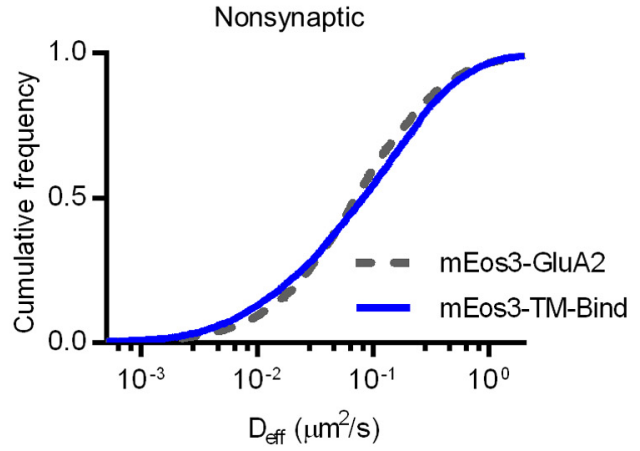

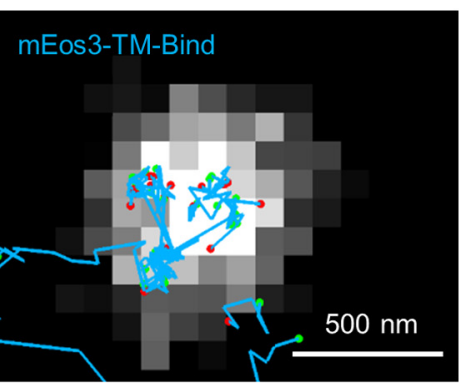

E

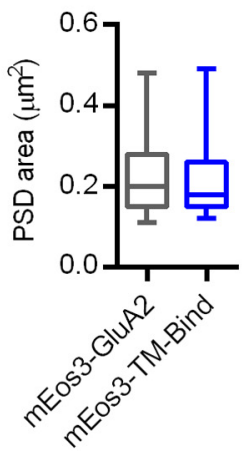

Figure 6. Diffusion of small-membrane protein and AMPAR within and near the excitatory synapse. A, Tracked motion of mEos-GluA2 (blue tracks) on PSDs marked by Homer1c-GFP. Green and red circles represent start and end, respectively, of individual tracks. $B$, Tracks of receptor (left) or smaller probe (right) on representative single PSDs marked by Homer1c-GFP. C, Cumulative frequency plot of synaptic diffusion coefficients ( $\mathrm{n}_{\text {mEos3-GluA2 }}=2065$ tracks/239 PSDs/13 neurons/2 cultures, $\mathrm{n}_{\mathrm{mEos}-\mathrm{TM}-\text { Bind }}=2403 / 284 / 12 / 2$ ). D, Cumulative frequency plot of nonsynaptic diffusion coefficients ( $\mathrm{n}_{\text {mEos3-GluA2 }}=3177$ tracks $/ 13$ neurons $/ 2$ cultures, $\left.\mathrm{n}_{\text {mEos3-TM-Bind }}=4044 / 12 / 2\right)$. E, Areas of Homer1C-GFP puncta that contained tracks $\left(\mathrm{n}_{\text {mEos3-GluA2 }}=239\right.$ PSDs, $\mathrm{n}_{\text {mEos3-TM-Bind }}=284$ ). C, ${ }^{* * * *} p<0.0001$ (Mann-Whitney U test). $\boldsymbol{D}, p=0.058$ (Mann-Whitney U test). E, $p=0.055$ (Mann-Whitney U test). C, D, $p<0.0001$ (K-S test).

slow overall mobility (e.g., differential access to percolation routes within the synapse). We cannot disentangle these possibilities based on experimental data. However, our modeling suggests that at least some of the difference may be due to steric effects.

\section{Mobility of small- and large-membrane proteins within the living synapse}

To obtain higher resolution of protein mobility within the synapse, we turned to single-molecule tracking PALM (smtPALM). We replaced the SEP in each probe with mEos3.1 (Zhang et al., 2012), a GFP that can be converted to red fluorescence with UV exposure. After cotransfection with Homer1c-GFP, weak UV irradiation prompted appearance of sparse, well-isolated, red molecules that could be tracked over time either within or outside the border of the synapse (Schneider et al., 2015). The average trajectory persisted for 6 frames before disappearing $(n=28,320$ tracks for all conditions in Fig. 6). Given the relative short length of tracks, we obtained the instantaneous, effective diffusion coefficient $\left(D_{\text {eff }}\right)$ as the best weighted linear fit of the MSD curve for every track that persisted for at least 4 frames (see Materials and Methods) as this method optimally separated distributions of nearly immobile molecules from more mobile ones ( $\mathrm{Lu}$ et al., 2014).

To determine whether the small-transmembrane probe and the large receptor diffuse differently within the synapse, we segregated the tracks into two groups. Tracks that were within the FWHM border of Homer1c-GFP puncta were considered synaptic; the remaining tracks were classified as nonsynaptic only if they were within the neuronal border and outside of dendritic clusters of unknown origin defined by a robust, automatic segmentation program, SR-Tesseler (Levet et al., 2015) (see Materials and Methods). A typical reconstruction of the individual moving mEos3-GluA2-containing AMPARs or mEos3-tagged membrane probe (Fig. 6A,B) showed that the displacements were heterogeneous inside individual synapses. The synaptic mEos3-GluA2 and mEos3-TM-Bind had identical distributions of $\mathrm{D}_{\text {eff }}<0.015 \mu \mathrm{m}^{2} / \mathrm{s}$ (Fig. $6 \mathrm{C}$ ), which suggests that a fraction of synaptic membrane proteins could be so tightly bound to scaffolds or tightly packed such that they could not diffuse distinguishably regardless of protein size. On the faster end of the diffusion spectrum $\left(>0.15 \mu \mathrm{m}^{2} / \mathrm{s}\right)$, the small-transmembrane probe diffused on average faster than the large receptor (Fig. $6 \mathrm{C}$ ), which suggests the existence of a subpopulation that can traverse the PSD more readily, consistent with partial synapse photobleaching results. The distribution of nonsynaptic $\mathrm{D}$ was in general shifted toward larger mobility compared with that of synaptic $\mathrm{D}$ for both proteins (Fig. $6 C, D$ ). The nonsynaptic $\mathrm{D}_{\text {eff }}$ of the AMPARs and the small binding probe had nearly identical distributions, and their medians were not different (Fig. 6D). The synaptic areas, as estimated by taking the FWHM of the Homerlc-GFP puncta that contained tracks, were not different on average for the two membrane proteins (Fig. 6E), suggesting that the difference in mobility is independent of the difference in synaptic size. Indeed, the averaged $D_{\text {eff }}$ within each PSD was not correlated with PSD size $\left(r^{2} \ll 0.001, p=\right.$ $0.91, n=239$ PSDs for mEos3-GluA2; $r^{2}=0.006, p=0.24$, $n=284$ mEos3-TM-Bind).

Together, results of simulation, partial bleaching, and singlemolecule tracking show that the small-transmembrane probe moves more freely than the AMPAR within the PSD. These observations provide support for the hypothesis that steric hindrance within the PSD can be a prominent factor that impedes the movement of transmembrane proteins, and suggests that the synapse selectively restricts protein distribution based on protein size. 


\section{PDZ-mediated binding was only partially responsible for stabilizing the synaptic mobility of the small-membrane probe}

To test directly the influence of binding to synaptic scaffold on the mobility of the membrane probe, we made a nonbinding mutant of the transmembrane probe (TM-Nonbind) by mutating threonine in the -2 position of the Stargazin C-tail to glutamate (T321E), a point mutation that has been shown by previous studies to abolish the binding between Stargazin and PSD-95 (Chetkovich et al., 2002; Choi et al., 2002). We examined fluorescence recovery $140 \mathrm{~s}$ after photobleaching (Fig. $7 A, B$ ) and also measured the near-equilibrium recovery within 52 min of photobleaching (Fig. 7C). The early recovery kinetics of nonbinding probe (SEP-TM-Nonbind) lay intermediate between the binding probe (SEP-TM-Bind) and the probe that lacked intracellular residues (SEP-TM) (Fig. $7 B, C$ ). At $30 \mathrm{~s}$ after bleaching the whole spine, the fluorescence of SEP-TM-Nonbind recovered less than that of SEP-TM and more than that of SEP-TM-Bind (mean \pm SEM: $50.1 \pm 2.4 \%$ TM, $33.0 \pm 3.1 \%$ TM-Nonbind, $20.0 \pm 1.8 \%$ TM-Bind; $n=28$ synapses/10 neurons TM, 27/3 TM-Nonbind, 42/11 TM-Bind; $p<0.0001$, ANOVA; Fig. 7D), suggesting that binding to PSD-95 is required for maximally stabilizing the mobility of fast exchanging receptors. At $140 \mathrm{~s}$, the spine fluorescence recovery extents of SEP-TM and SEP-TM-Nonbind were not different (mean \pm SEM: $73.8 \pm 3.5 \% \mathrm{TM}, 67.8 \pm 3.1 \%$ TM-Nonbind; $p=0.23$ ), but they were both higher than that of SEP-TM-Bind $(38.5 \pm 3.0 \%$; $p<0.0001$ compared with TM or TM-Nonbind; Fig. $7 E$ ). At $52 \mathrm{~min}$, the recovered spine fractions of all three probes were not statistically different (Fig. $7 F$ ), indicating that a single PDZ binding motif is not sufficient to regulate the relative proportions of mobile and immobile TM proteins in the spine. The fluorescence recovery of all three probes was not different following photobleaching the dendritic shaft (mean \pm SEM: $67.1 \pm 2.1 \%$ TM, $73.5 \pm 3.8 \%$ TM-Nonbind, $75.2 \pm 3.4 \%$ TM-Bind; $n=21$ dendritic shaft segments/9 neurons TM, 18/3 TM-Nonbind, 27/9 TM-Bind; $p=0.18$; Fig. 7G).

The spine enrichment of SEP-TM-Bind was the highest of the three probes, essentially identical to Homer 1C, whereas SEP-TM and SEP-TM-Nonbind were strikingly less enriched and not different from one another (Fig. 7I). Thus, the PDZ-binding motif was necessary and sufficient for accumulating the TM probe in the spine. The PSD areas of the photobleached spines were not different for all three probes (Fig. $7 \mathrm{H}$ ). The recovered fraction of SEP-TM-Bind in individual spines was negatively correlated with the size of the synapse at 30 and $140 \mathrm{~s}$, although the correlation was weak as the variation in synapse size could only explain 15\%$17 \%$ of the variation in recovery; there was no correlation at 52 min after bleaching (Table 2). There was no correlation between the recovered fraction and synapse size for SEP-TM and SEPTM-Nonbind at any time after bleaching (Table 2). In addition, there was no correlation between the recovered fraction and spine enrichment for any of the three probes (Table 2). Together, these results indicate that the reduction of TM probe mobility by the addition of the nonbinding Stg C-tail did not arise due to a difference in synapse sizes or in concentration gradients of molecules between the spine head and dendrite but instead reflects the effect of the added intracellular bulk.

Kinetics of recovery after photobleaching an entire spine are influenced not only by diffusion within the synapse but also by mobility on the spine head and dynamics of transit across the spine neck to the dendritic shaft. To measure directly the diffusion of TM probes in the synapse, we applied smtPALM (Fig. $7 J$ ). Outside the synapse, the three TM probes showed nearly identical diffusion distributions (Fig. $7 K$, right), consistent with the notion that TM protein diffusion is only weakly dependent on protein size in the relative absence of obstacles. However, within the synapse, mEos3-TM-Nonbind diffused faster than mEos3-TM-Bind but more slowly than mEos3-TM (Fig. $7 K$, left). This indicates that, although PDZ-mediated binding can limit protein motion in the synapse, it is not the only factor in stabilizing the synaptic diffusion of the membrane probe.

As it could be the case that introducing exogenous PSD-95binding motifs could alter the interior landscape of the PSD and thus indirectly influence diffusion of the expressed probe, we examined PSD-95 distribution using PALM (MacGillavry et al., 2013). We knocked down endogenous PSD-95, rescued expression with a knockdown-resistant PSD-95-mEos2 (MacGillavry et al., 2013) (Fig. 7L), and coexpressed in the same neurons one of the three SEP-tagged probes. After 3-4 d of coexpression, the nano-organization of PSD-95 (i.e., PSD area, nanodomain size, and molecular density within nanodomain) was on average not different for cells expressing any of the three probes (Fig. $7 \mathrm{M}, N$ ). Together, these results support a model in which protein-protein binding is not the only factor in stabilizing the synaptic diffusion of transmembrane proteins.

\section{Intracellular protein bulk can influence the synaptic mobility of the small-transmembrane probe}

The PSD consists of a large number of high molecular weight proteins (Husi et al., 2000; Sheng and Hoogenraad, 2007; Burette et al., 2012), which include submembranous scaffold proteins typified by PSD-95. The dense assembly of these scaffolding proteins could sterically interact with transmembrane proteins like receptors through their cytoplasmic domains. However, it is unclear whether and how steric hindrance proximal to the inner face of the postsynaptic membrane influences protein diffusion. To examine the effect of intracellular protein bulk on the mobility of the membrane probe, we fused the GFP variant Cerulean 3 to the cytoplasmic terminus of the TM probe (SEP-TM-Cerulean) and examined recovery after photobleaching spines. SEP-TMCerulean and SEP-TM-Nonbind recovered similarly slower than SEP-TM at $30 \mathrm{~s}$ after bleaching (Fig. $8 A, B, D$ ), indicating that intracellular protein bulk can influence the lateral mobility of a TM protein on the spine surface membrane. Near the equilibrium state, the recovered fractions of the three probes were not different in the spine (Fig. $8 C, E, F$ ) or dendrite (Fig. 8G), indicating, as expected, that the reduced mobility was not associated with lower fractional exchange, but an increased retention time in the synapse. The PSD areas and the spine enrichment of the three probes in the photobleached spines were not different (Fig. $8 \mathrm{H}, \mathrm{I})$; and importantly, there was no correlation between the recovered spine fraction and either the synapse size or spine enrichment for any of the three probes (Table 2). Together, these results indicate that TM protein mobility is reduced by added intracellular bulk. This is not due to a difference in synapse size or an altered concentration gradient between the spine head and its parent dendrite, but most likely arises from reduced diffusibility in the crowded environment of the synapse.

To examine this directly, we applied smtPALM on cells expressing each of the mEos3-tagged probes while coexpressing a PSD-marking protein Homer1c-GFP (Fig. 8J). Within the synapse, both mEos3-TM-Nonbind and mEos3-TM-Cerulean diffused slower than mEos3-TM (Fig. $8 \mathrm{~K}$, left). Interestingly, the effect of added Cerulean was not as great as that of the nonbinding Stg C-tail, which suggests that factors other than total bulk can influence mobility within the synapse, perhaps tertiary struc- 

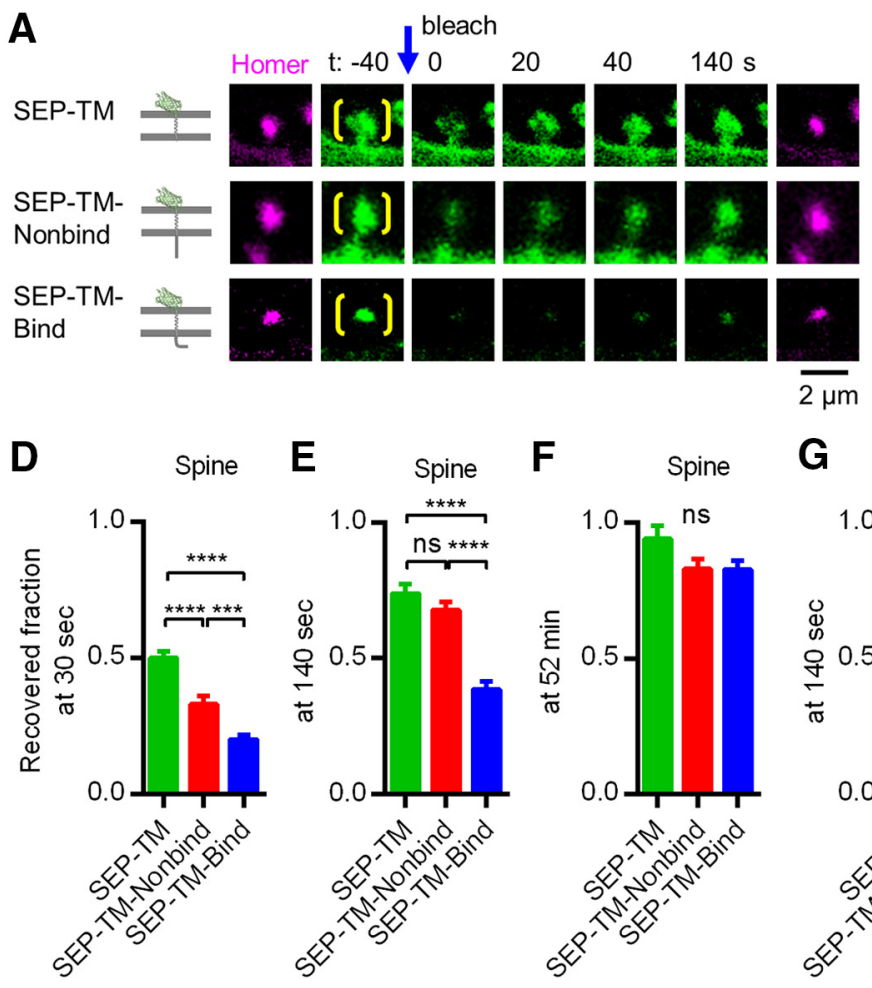

F Spine

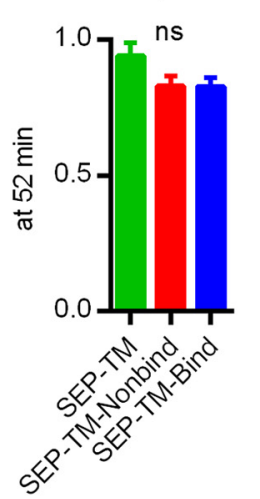

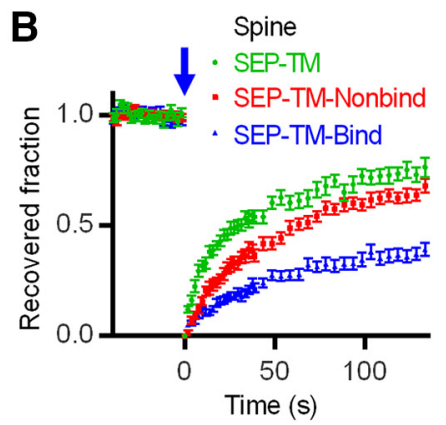

G Dendrite

H
C Spine

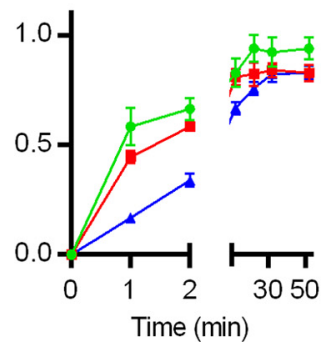

I
J

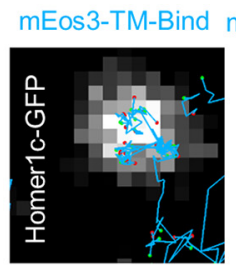

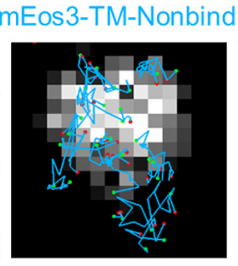

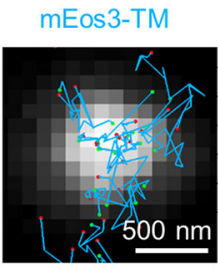

K

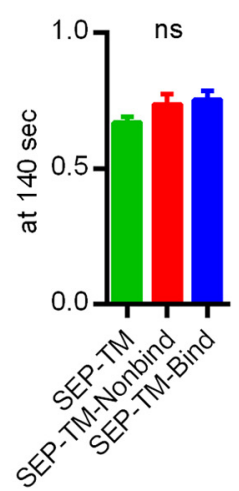

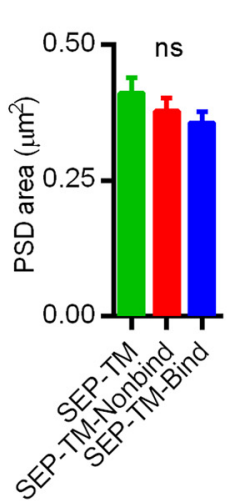
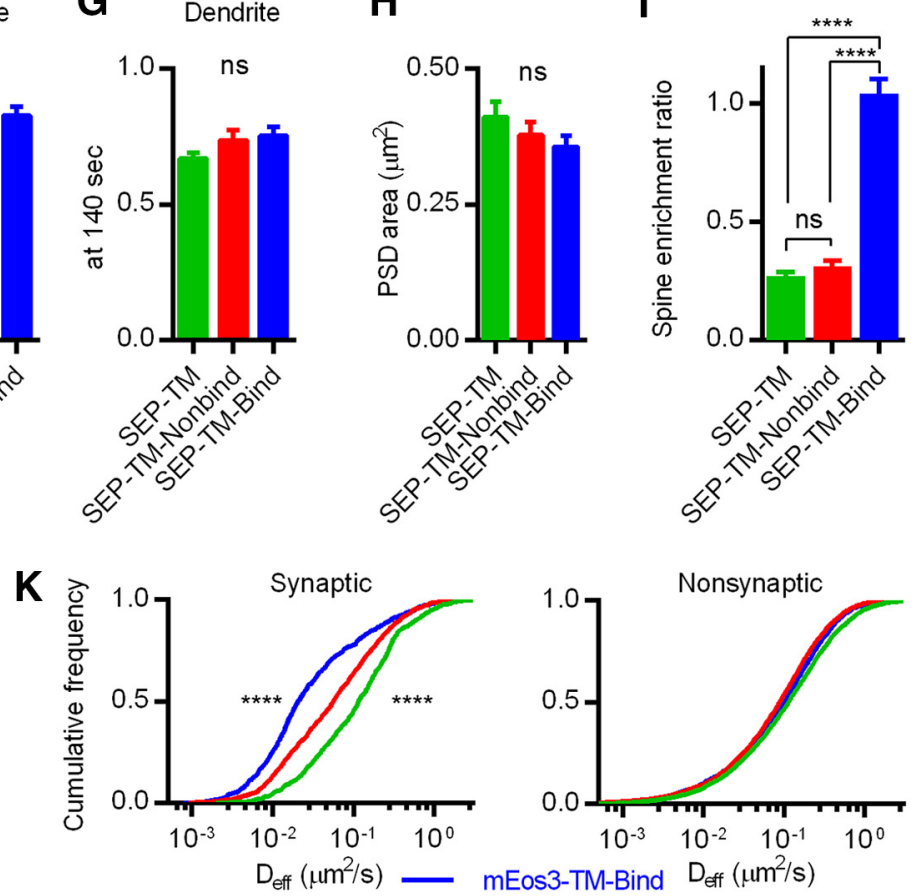

$\mathbf{L}$

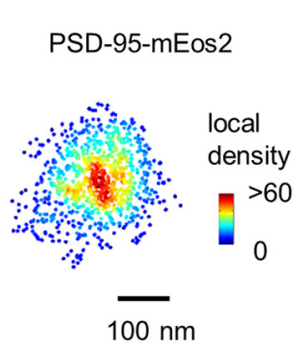

M

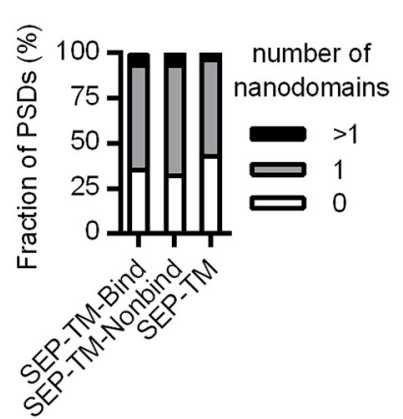

$\mathbf{N}$

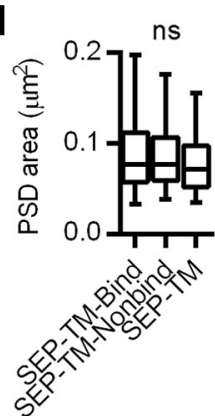

\section{mEos3-TM-Nonbind \\ mEos3-TM}

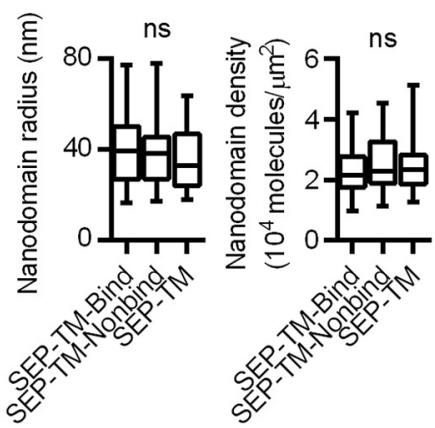

Figure 7. PDZ-mediated binding is partly responsible for stabilizing the synaptic mobility of a small-membrane probe. $A$, Representative examples of FRAP in spines of neurons coexpressing Homer1c-mCherry and one of the SEP-tagged transmembrane probes: TM, TM-Nonbind, or TM-Bind. B, Recovery curves of $140 \mathrm{~s}$ FRAP on spines (SEP-TM, $n=28$ ROIs/10 neurons/2 cultures; SEP-TM-Nonbind, 27/7/2; SEP-TM-Bind, 42/11/2). C, Recovery curves of 52 min FRAP on spines (SEP-TM, $n=12$ ROIs/3 neurons/3 cultures; SEP-TM-Nonbind, 14/3/3; SEP-TM-Bind, 19/3/3). D-F, Recovered fractions in the spine at $30 \mathrm{~s}(\boldsymbol{D}), 140 \mathrm{~s}(\boldsymbol{E})$, and $52 \mathrm{~min}(\boldsymbol{F})$. G, Recovered fractions in the dendrite at $140 \mathrm{~s}$ (SEP-TM, $n=25$ ROIs/10 neurons/2 cultures; SEP-TM-Nonbind, 23/7/2; SEP-TM-Bind, 27/11/2). The (H) PSD areas and $(\boldsymbol{I})$ spine enrichment in spines that were targeted for bleaching in $\boldsymbol{B}$ and $\boldsymbol{C}$. $\boldsymbol{J}$, Representative smtPALM tracks (cyan segments; green and red dots indicate where localizations appeared and disappeared) superimposed on individual synapses marked by Homer 1 C-GFP. $K$, Cumulative frequency plots of instantaneous diffusion coefficients $\left(D_{\text {eff }}\right)$ inside Homer1c-GFP-marked synapses (left, mEos3-TM-Bind, $n=918$ tracks/132 PSDs/10 neurons/2 cultures; mEos3-TM-Nonbind, 1269/178/10/2; mEos3-TM, 686/180/13/2) and outside synapses (right, mEos3-TM-Bind, $n=3553$ tracks; mEos3-TM-Nonbind, 6247; mEos3-TM, 6156). Statistics on synaptic $\mathrm{D}_{\text {eff }}$. K-S test and Mann-Whitney U test. ${ }^{* * * *} p<0.0001$, mEos3-TM-Bind versus mEos3-TM-Nonbind, or mEos3-TM-Nonbind versus mEos3-TM. L, Representative nanoscale map of mEos2-tagged shrPSD-95 coexpressed with one of the three probes: SEP-TM-Bind, SEP-TM-Nonbind, and SEP-TM. Local density calculated as in MacGillavry et al. (2013). $\boldsymbol{M}$, Relative proportion of PSDs with 0, 1, or more PSD-95 nanodomains in cells expressing SEP-TMBind, SEP-TM-Nonbind, or SEP-TM (SEP-TM-Bind, $n=110$ PSDs/8 neurons/2 cultures; SEP-TM-Nonbind, 59/7/2; SEP-TM, 128/5/2). , PSD area, PSD-95 nanodomain radius, and PSD-95 molecular density in nanodomain (SEP-TM-Bind, $n=110$ PSDs/79 nanodomains; SEP-TM-Nonbind, 59/44; SEP-TM-Bind, 128/79). Kruskal-Wallis ANOVA: ns, Not significant ( $p=0.23$ for PSD area, $p=0.35$ nanodomain radius, and $p=0.24$ nanodomain density). $\boldsymbol{D}-\boldsymbol{I}$, One-way ANOVA $(p<0.0001(\boldsymbol{D}, \boldsymbol{E}, \boldsymbol{I})$. ns, Not significant. $\boldsymbol{F}, p=0.10 . \mathbf{G}, p=0.18$. $\boldsymbol{H}, p=0.27)$. Bonferroni-corrected post hoc pairwise comparisons $t$ test: ${ }^{* * *} p<0.0001(\boldsymbol{D}, \boldsymbol{E}, \boldsymbol{I}) ;{ }^{* * *} p=0.0004(\boldsymbol{D})$; ns, Not significant, $p=0.68(\boldsymbol{E}) ; \mathrm{ns}, p>0.99(\boldsymbol{I})$. 
Table 2. Correlation between FRAP and either PSD area or spine enrichment ratio of various TM probes ${ }^{a}$

\begin{tabular}{|c|c|c|c|c|c|c|}
\hline \multirow[b]{2}{*}{ Probe } & \multicolumn{3}{|c|}{ Recovered fraction (t) versus PSD area } & \multicolumn{3}{|c|}{ Recovered fraction (t) versus spine enrichment } \\
\hline & $30 s$ & $140 \mathrm{~s}$ & $52 \mathrm{~min}$ & $30 s$ & $140 s$ & $52 \mathrm{~min}$ \\
\hline \multirow[t]{2}{*}{ SEP-TM } & $\mathrm{ns}, p=0.10$ & $\mathrm{~ns}, p=0.14$ & $\mathrm{~ns}, p=0.27$ & $\mathrm{~ns}, p=0.92$ & $\mathrm{~ns}, p=0.19$ & $\mathrm{~ns}, p=0.095$ \\
\hline & $n=62 / 15 / 5$ & $n=62 / 15 / 5$ & $n=12 / 3 / 3$ & $n=62 / 15 / 5$ & $n=62 / 15 / 5$ & $n=12 / 3 / 3$ \\
\hline \multirow[t]{2}{*}{ SEP-TM-Nonbind } & $\mathrm{ns}, p=0.46$ & $\mathrm{~ns}, p=0.76$ & $\mathrm{~ns}, p=0.38$ & $\mathrm{~ns}, p=0.24$ & $\mathrm{~ns}, p=0.44$ & $\mathrm{~ns}, p=0.12$ \\
\hline & $n=46 / 11 / 5$ & $n=46 / 11 / 5$ & $n=14 / 3 / 3$ & $n=46 / 11 / 5$ & $n=46 / 11 / 5$ & $n=14 / 3 / 3$ \\
\hline \multirow[t]{3}{*}{ SEP-TM-Bind } & $p=0.011$ & $p=0.0064$ & $\mathrm{~ns}, p=0.66$ & $\mathrm{~ns}, p=0.16$ & $\mathrm{~ns}, p=0.92$ & $\mathrm{~ns}, p=0.66$ \\
\hline & $r=-0.39$ & $r=-0.41$ & $n=19 / 3 / 3$ & $n=42 / 11 / 2$ & $n=42 / 11 / 2$ & $n=19 / 3 / 3$ \\
\hline & $n=42 / 11 / 2$ & $n=42 / 11 / 2$ & & & & \\
\hline \multirow[t]{2}{*}{ SEP-TM-Cerulean } & $\mathrm{ns}, p=0.074$ & $\mathrm{~ns}, p=0.11$ & $\mathrm{~ns}, p=0.58$ & $\mathrm{~ns}, p=0.17$ & $\mathrm{~ns}, p=0.25$ & $\mathrm{~ns}, p=0.57$ \\
\hline & $n=43 / 6 / 3$ & $n=43 / 6 / 3$ & $n=17 / 3 / 3$ & $n=43 / 6 / 3$ & $n=43 / 6 / 3$ & $n=17 / 3 / 3$ \\
\hline
\end{tabular}

${ }^{a}$ Statistics of linear regression analyses for recovered fraction at various time points and either PSD area or spine enrichment ratio of various TM probes. ns, Not significant. Sample number $n$ is shown in number of spines/neurons/cultures.

A
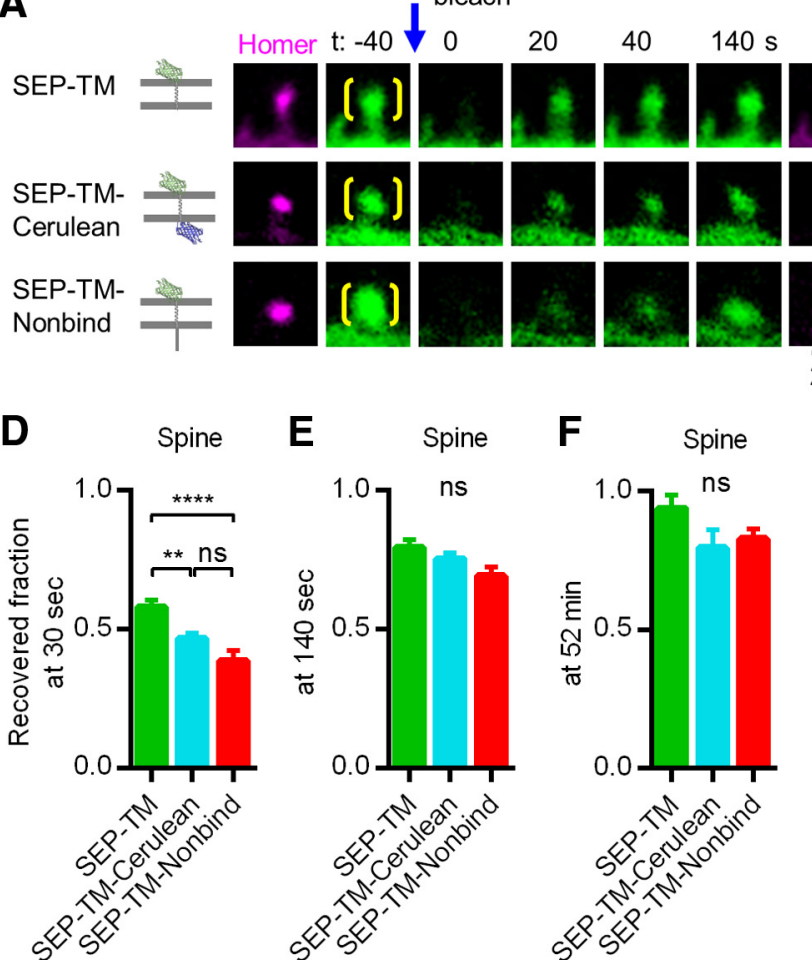

J
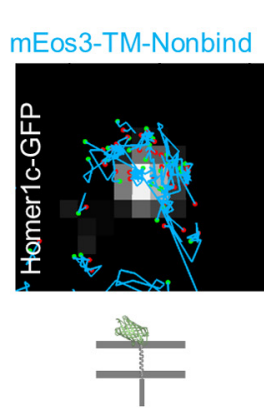

E Spine

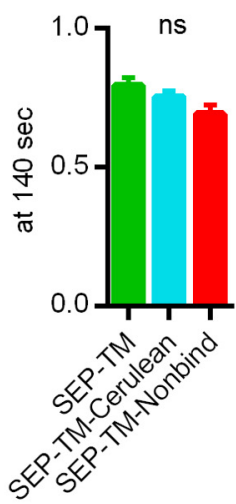

F Spine
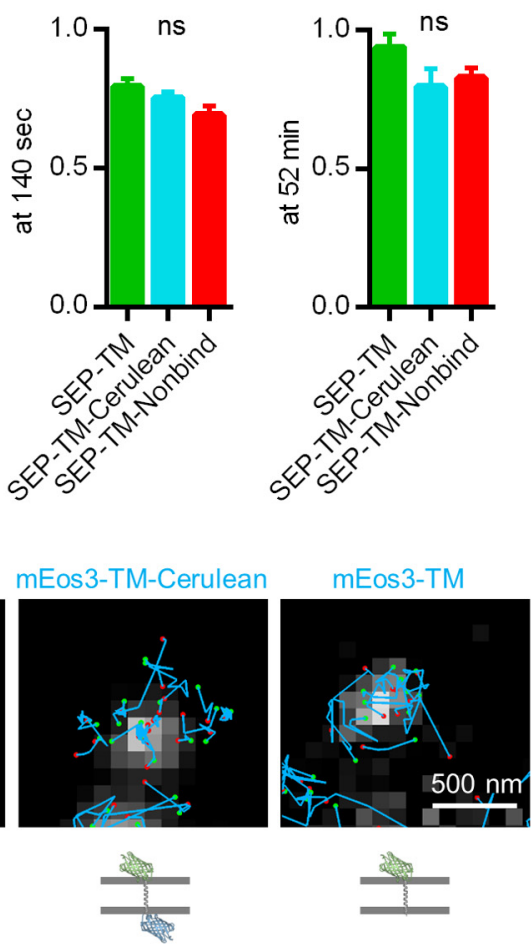

B

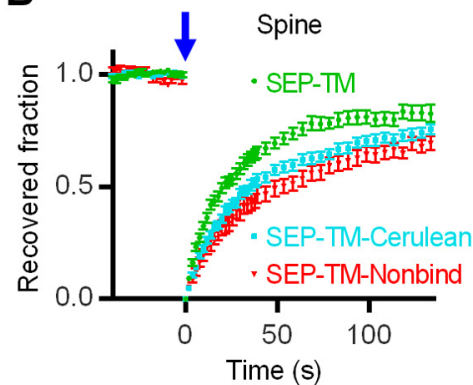

G Dendrite $\mathbf{H}$

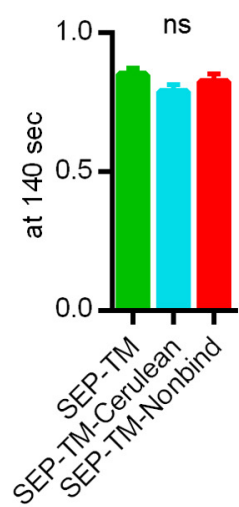

H I

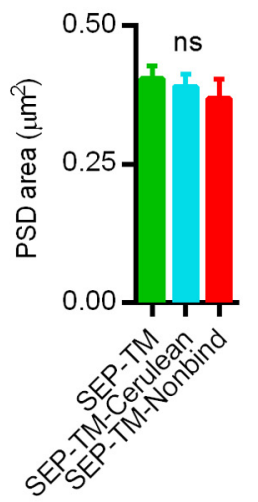

C

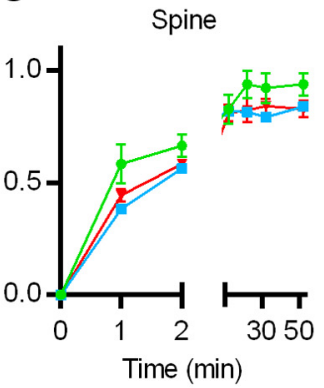

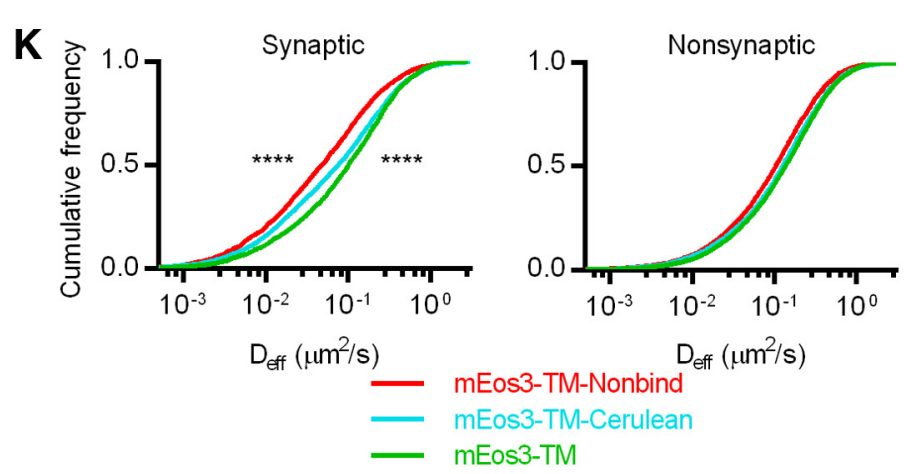

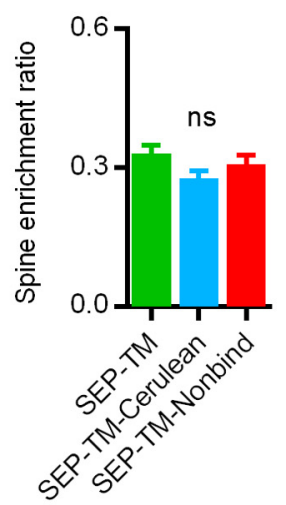

Figure 8. Intracellular protein bulk can influence the mobility of small-membrane protein. $A$, Representative examples of FRAP in spines of neurons coexpressing Homer1c-mCherry and one of the SEP-tagged transmembrane probes: TM, TM-Cerulean, or TM-Nonbind. B, Recovery curves of $140 \mathrm{~s}$ FRAP on spines (SEP-TM, $n=34$ ROIs $/ 5$ neurons $/ 3$ cultures; SEP-TM-Cerulean, $43 / 6 / 3$, SEP-TM-Nonbind 19/4/3). C, Recovery curves of $52 \mathrm{~min}$ FRAP on spines (SEP-TM, $n=12$ ROls $/ 3$ neurons $/ 3$ cultures; SEP-TM-Cerulean, 17/3/3; SEP-TM-Nonbind, 14/3/3). D-F, Recovered fractions in the spine at $30 \mathrm{~s}(\boldsymbol{D}), 130 \mathrm{~s}(\boldsymbol{E})$, and $52 \mathrm{~min}(\boldsymbol{F})$. $\boldsymbol{G}$, Recovered fractions in the dendrite at $140 \mathrm{~s}$ (SEP-TM, $n=34$ ROIs $/ 5$ neurons/3 cultures; SEP-TM-Cerulean, 34/6/3; SEP-TM-Nonbind, 15/4/3). Areas of $(\boldsymbol{H})$ Homer1c-mCherry puncta and $(\boldsymbol{I})$ spine enrichment in spines that were targeted for bleaching. J, Representative smtPALM tracks (cyan segments; green and red dots indicate where localizations appeared and disappeared) superimposed on individual synapses marked by Homer1c-GFP. $\boldsymbol{K}$, Cumulative frequency plots of instantaneous diffusion coefficients ( $\left.\mathrm{D}_{\text {eff }}\right)$ inside Homer1cGFP-marked synapses (left, mEos3-TM-Nonbind, $n=3283$ tracks/423 PSDs/14 neurons/3 cultures; mEos3-TM-Cerulean, 3645/602/20/3; mEos3-TM, 2500/265/10/3) and outside synapses (right,

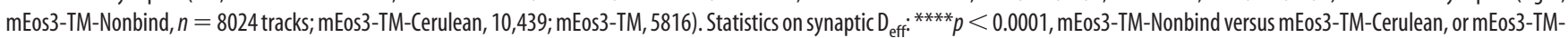
Cerulean versus mEos3-TM (K-S test and Mann-Whitney U test). $\boldsymbol{D}-\mathbf{I}$, One-way ANOVA $(\boldsymbol{D}, p<0.0001$. ns, Not significant. $\boldsymbol{E}, p=0.054 . \boldsymbol{F}, p=0.24 . \mathbf{G}, p=0.92 . \boldsymbol{H}, p=0.53 . \boldsymbol{I}, p=0.26) . \boldsymbol{D}$, Bonferroni-corrected post hoc pairwise comparisons $t$ test ${ }^{* * * *} p<0.0001{ }^{* *} p=0.0028$; ns, Not significant, $p=0.16$ ). 
A

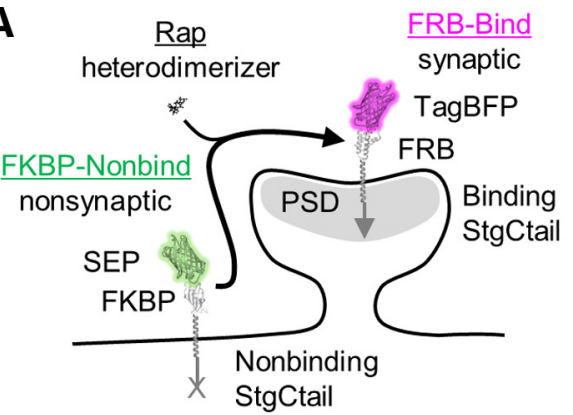

C

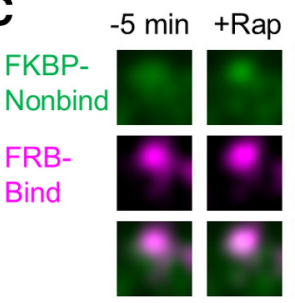

E

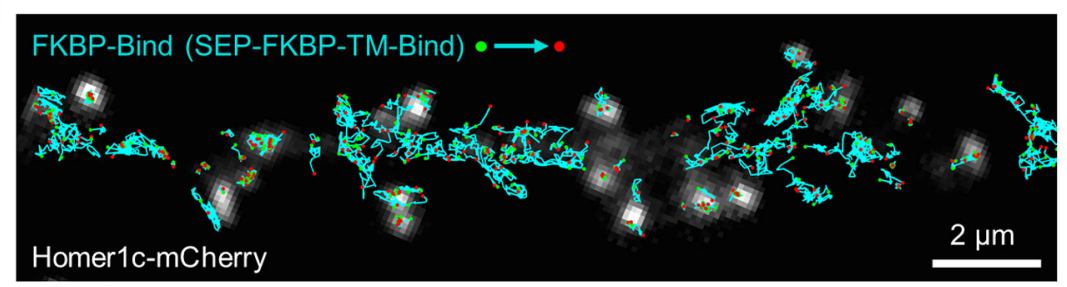

$\mathbf{F}$

FKBP-Bind

(B)

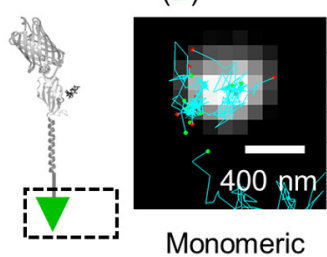

Monovalent binding

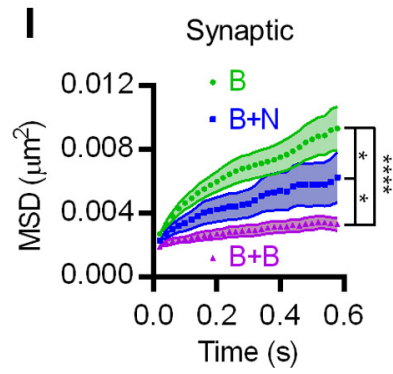

G FKBP-Bind + FRB-Nonbind $(\mathrm{B}+\mathrm{N})$

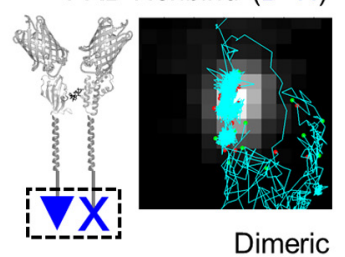

Monovalent binding
H FKBP-Bind + FRB-Bind $(B+B)$

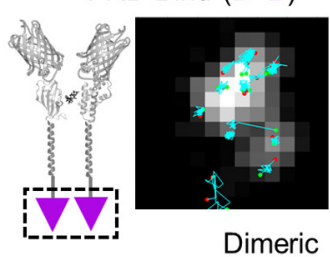

Divalent binding
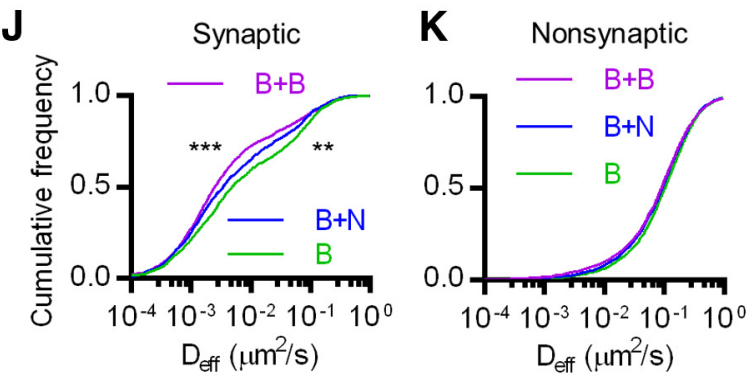

Figure 9. Divalent PDZ-binding motifs can stabilize TM proteins within the synapse more than monovalent binding can. $\boldsymbol{A}$, Diagram illustrating the strategy to induce dimerization and translocation of TM probes into dendritic spines using Rapalog (Rap). B, A DIV 16 hippocampal neuron expressing SEP-FKBP-TM-Nonbind (FKBP-Nonbind) and TagBFP-FRB-TM-bind (FRB-Bind) for $2 \mathrm{~d}$ before (Pre), and 40 min after (Post) bath incubation with $1 \mu \mathrm{m}$ Rap. C, Time-lapse of the highlighted region drawn in $\boldsymbol{B}$. D, Enrichment of FKBP-Nonbind (mean \pm SEM) at dendritic spines containing FRB-Bind ( $n=34$ spines $/ 3$ fields $/ 3$ cultures for Rapalog, 28/3/3 vehicle) before and after Rapalog or vehicle ( $0.2 \%$ ethanol) application. $\boldsymbol{E}$, The first (green) and last (red) localized positions of FKBP-Bind (SEP-FKBP-TM-Bind) molecules tracked for $\geq 8$ frames, superimposed on a typical dendritic segment coexpressing Homer1 1 -mCherry as a synaptic marker. $\boldsymbol{F}$, Diagram representing the FKBP-Bind the monomeric binding probe (left), and typical tracks in synapses coexpressing this probe (B) and Homer1c-mCherry (right). G, Diagram representing the FKBP-Bind and FRB-Nonbind (TagBFP-FRB-TM-Nonbind) dimerized into a monovalent binding probe in the presence of Rapalog (left), and typical synaptic tracks in cells coexpressing these two probes $(B+N)$ and Homer1c-mCherry (right). $\boldsymbol{H}$, Diagram representing the FKBP-Bind and FRB-Bind (TagBFP-FRB-TM-Bind) dimerized into a divalent binding probe in the presence of Rapalog (left), and typical synaptic tracks in cells coexpressing these two probes $(B+B)$ and Homer1c-mCherry (right). $I$, MSD over time within synapses ( $n=352$ tracks $/ 3$ cultures for B, 174/3 B $+N, 251 / 3 B+B)$. Kruskal-Wallis ANOVA $(p<0.0001)$ post hoc comparisons

ture. Outside of the synapse, the three probes exhibited nearly identical distributions of $\mathrm{D}_{\text {eff }}$ (Fig. $8 \mathrm{~K}$, right). Together, these results indicate that the bulk of the intracellular domain alone can influence the mobility of a transmembrane protein within the synapse, consistent with the notion that dense and irregular environment of the PSD provides substantial steric hindrance to the movement of receptors and other synaptic TM proteins.

\section{Divalent PDZ-binding interactions} within the synapse can stabilize the mobility of transmembrane proteins more than monovalent interactions can AMPA receptors could bear multiple TARPs capable of binding PSD scaffold molecules (Hastie et al., 2013). To test the role of divalent receptor-scaffold interactions on receptor mobility in the synapse, we developed a chemically inducible heterodimerization strategy that would permit us to acutely convert our typical monovalent binding probe to one with divalent PDZ-binding capacity. To do this, we added to our TM probes either FKBP or FRB, protein domains that can be crosslinked via the small molecule rapalog (Fig. 9A). By crosslinking one binding probe to a second probe either carrying a second binding motif or not, the additional effect of divalent binding could be assessed as well as distinguished from the effect of added bulk. To avoid potential offtarget effects of lengthening the binding C-tail via insertion in the cytoplasmic domain, we inserted the dimerization motifs in the extracellular domain and created four fusion proteins: SEP-FKBP-TM-Bind (FKBP-Bind), SEP-FKBP-TM-Nonbind (FKBP-Nonbind), TagBFP-FRB-TM-Bind (FRB-Bind), and TagBFP-FRB-TM-Nonbind (FRB-Nonbind).

We tested the dimerization assay in neurons coexpressing FKBP-Nonbind and FRB-Bind. In the absence of rapalog, FKBP-Nonbind was on average $37 \%$ as enriched as FRB-Bind in spines; incubation with $1 \mu \mathrm{M}$ rapalog resulted in an increase in the spine enrichment of FKBP-

by Mann-Whitney $U$ test. ${ }^{*} p=0.0194$ ( $B$ vs $\left.B+N\right), 0.0395$ $(B+N$ vs $B+B) .{ }^{* * *} p<0.0001$ ( $B$ vs $B+B$ ). J, Cumulative frequency distributions of $D_{\text {eff }}$ within synapses (left; $n=1982$ tracks/231 PSDs/7 cells/3 cultures for $B+B, 868 / 201 / 9 / 3$ $\mathrm{B}+\mathrm{N}, 1415 / 273 / 7 / 3 \mathrm{~B})$. Kruskal-Wallis ANOVA $(p<0.0001)$ post hoc comparisons by $K-S$ test. ${ }^{* * *} p=0.0004$ ( $B+B$ vs $B+N) .{ }^{* *} p=0.0021(B+N$ vs $B)$; and by Mann-Whitney $U$ test $p=0.0053(\mathrm{~B}+\mathrm{B}$ vs $\mathrm{B}+\mathrm{N}), p=0.0003(\mathrm{~B}+\mathrm{N}$ vs $\mathrm{B}) . \boldsymbol{K}$, Cumulative frequency distributions of $D_{\text {eff }}$ outside of synapses (right; $n=3621$ tracks for $\mathrm{B}+\mathrm{B}, 3465 \mathrm{~B}+\mathrm{N}, 5750 \mathrm{~B}$ ). 
Nonbind, which plateaued on average to $79 \%$ after $40 \mathrm{~min}$, whereas incubation with the vehicle resulted in no change (Fig. $9 B-D)$. Thus, rapalog can induce dimerization between TM probes containing extracellular FKBP and FRB domains.

We then cotransfected three groups of cultured neurons, obtaining distinct populations expressing only FKBP-Bind (a monomeric binding probe) (Fig. 9F, diagram), FKBP-Bind and FRB-Nonbind (which can dimerize to form a monovalent binding probe) (Fig. 9G, diagram), or FKBP-Bind and FRB-Bind (which can dimerize to form a divalent binding probe) (Fig. $9 \mathrm{H}$, diagram). To measure the surface mobility of the probes, we used uPAINT single-molecule tracking of fluorescently conjugated nanobodies recognizing SEP but not TagBFP (Fig. 9E). We measured the MSD and effective diffusion coefficients of FKBP-Bind in these groups of neurons after a 1 to $2 \mathrm{~h}$ preincubation with 1 $\mu \mathrm{M}$ rapalog (Fig. $9 F-H$, right). The $\mathrm{D}_{\text {eff }}$ distributions of these three probes outside of the synapse were nearly identical (Fig. $9 K)$, consistent with the notion that diffusion coefficient is only weakly dependent on particle size in membrane regions with few obstacles. Within the crowded environment of the synapse, however, they behaved differently. The monomeric probe explored a larger area (Fig. 9I) and diffused faster (Fig. 9J) in synapses than both dimeric probes, indicating that dimerization even without adding an extra receptor-scaffold binding motif can reduce TM protein mobility within the synapse. As expected, the divalent binding probe explored even less area (Fig. 9I) and more slowly (Fig. $9 J$ ) than the dimeric but monovalent binding probe, indicating that polyvalent receptor-scaffold binding interactions can confine TM proteins more effectively than monovalent binding can within the synapse. Together, these results support the hypothesis that both steric and binding interactions can stabilize the mobility of TM proteins within the synapse.

\section{Discussion}

Combining simulations and live-cell imaging methods, we examined the influence of protein-protein binding and of protein steric hindrance within the postsynaptic density on the mobility of synaptic membrane proteins. Measurements of molecular mobility across the lateral extent of the synapse and dendritic spines revealed that the dynamics of a receptor-like TM probe strongly depended on two features of the intracellular domain: the presence of protein bulk and the capacity for protein-protein binding. By simulating receptor diffusion within a model incorporating the measured positions of the critical postsynaptic scaffold macromolecule PSD-95, our simulations suggest that the nanoscale heterogeneous organization of PSD-95 alone can alter the dwell time of synaptic receptors or any other intracellularly bulky membrane protein. We propose that the nanoscale organization of scaffold proteins in the PSD shapes the mobility and distribution of synaptic membrane proteins through two mechanisms: steric effects and establishing the position and density of binding partners in the synapse. These steric effects will depend on the size of the diffusing protein. Overall, the interplay between these mechanisms will likely regulate the spatial distribution and dynamics not just of neurotransmitter receptors, but of numerous critical transmembrane proteins, such as ion channels and adhesion molecules.

A previous study has suggested that the PSD could act as a size-exclusion environment akin to the synaptic cleft, demonstrated by using lipids tagged by differently sized bulks in the extracellular domain (Renner et al., 2009a). Our results extend this picture of the sieve-like synapse in several key ways. First, they demonstrate that this idea applies to transmembrane pro- teins. Second, they indicate that the larger the intracellular bulk of the membrane protein, the smaller the synaptic area it could explore. This was demonstrated by simulations and deduced from photobleaching within individual synapses that showed a size dependence in protein mobility. Finally, they suggest that the dense crowding of synaptic scaffolding proteins limits the escape of mobile membrane proteins from the PSD (and consequently their incorporation as well). This was demonstrated in simulations, which showed that PSDs modeled with the measured distributions of scaffolding proteins retained receptors longer than those with randomized distributions. More support of this notion comes from the experimental results that added intracellular bulk slowed down the exchange of the small TM probe.

It is well known that the PDZ-mediated binding motif of Stargazin plays a prominent role in stabilizing the mobility of AMPARs (Chen et al., 2000; Bats et al., 2007; Sainlos et al., 2011), and here we showed that it could also stabilize even minimal synthetic transmembrane proteins. However, our results further demonstrated that intracellular bulk could have substantial influence on the lateral mobility of transmembrane proteins, as well as their positioning within subdomains of the synapse. This influence may have significant impact on excitatory synaptic transmission in diverse situations. In particular, the physical bulk of an AMPAR complex is ultimately determined by multiple classes of proteins (Tomita et al., 2003; Cho et al., 2007; Milstein et al., 2007; Schwenk et al., 2009; Soto et al., 2009; Kalashnikova et al., 2010; von Engelhardt et al., 2010) that additionally associate with the GluA tetramer. Indeed, biochemically isolated, native AMPAR complexes are 20\%-120\% larger than the GluA tetrameric core (Schwenk et al., 2012). We speculate that differently sized AMPAR subcomplexes can have dramatic impact on function not only due to their diverse binding motifs and channel gating kinetics, but also because of their differences in protein bulk.

Given this, it is possible that a significant fraction of synaptic receptors that remain following disruption to receptor-scaffold binding (Bats et al., 2007; Sainlos et al., 2011) are retained in the synapse by steric effects. In an interesting further example, desensitization-driven dissociation of AMPARs from Stargazin (Tomita et al., 2004; Constals et al., 2015) not only alters binding to PSD-95 but also reduces their size. Each of these effects may contribute to the observed increase in mobility of desensitized receptors (Constals et al., 2015). Together, these considerations suggest that protein-size-dependent regulation of protein mobility and dwell times could be a mechanism regulating the synaptic distribution of AMPARs.

A notable aspect of steric interactions is that they are agnostic to molecular identity, and so will influence synaptic distribution of ion channels, adhesion proteins, or any transmembrane protein. A recent study showed that synaptic accumulation of receptors can occur regardless of the type of receptor (Granger et al., 2013). It is tempting to speculate that macromolecular crowding offers a mechanism to explain how the synapse can retain TM proteins, regardless of specific protein-protein binding motifs. Molecular crowding alone cannot account for the synaptic enrichment of receptors but, in combination with binding, presents an efficient way to accumulate and retain membrane proteins at the synapse (Holcman and Triller, 2006; Haselwandter et al., 2011; Masson et al., 2014). Thus, we suggest a two-step process that can distribute receptors throughout the synapse interior. TM proteins first need binding partners to accumulate at the periphery of the PSD (Bats et al., 2007; Opazo and Choquet, 2011). 
Indeed, we found that a PDZ-binding motif was necessary and sufficient to drive spine enrichment of the TM probes. Second, even proteins that have unbound from scaffolds can be nonselectively incorporated to the interior by the minute-scale morphing of the PSD (Blanpied et al., 2008; Kerr and Blanpied, 2012) as well as molecular positional dynamics and exchange of synaptic scaffolding proteins (Gray et al., 2006; MacGillavry et al., 2013; Chazeau et al., 2014).

Our simulations assumed that, other than the mobile probe molecules, PSD-resident proteins were held in stable positions. Consistent with this assumption, the turnover of endogenous PSD-95 at the synapse is extremely slow, as FRAP of PSD-95 bearing a Venus tag in a knock-in mouse exchanges only $\sim 10 \%$ in $60 \mathrm{~min}$ (Fortin et al., 2014). Exchange of overexpressed PSD-95 is elevated compared with this but still slow, with time constants of 30-100 min in the hippocampus (Gray et al., 2006; Sharma et al., 2006; Blanpied et al., 2008), depending on age (Gray et al., 2006). These observations suggest that, within the short time frame of our simulations, such exchange will be of minimal impact and PSD-95 can be considered immobile. However, ongoing spine actin polymerization alters PSD morphology over minutes (Kuriu et al., 2006; Kerr and Blanpied, 2012; Ziv and Fisher-Lavie, 2014), the nanoscale organization of the PSD is temporally dynamic (Nair et al., 2013) and activity-dependent (MacGillavry et al., 2013), and single-molecule tracking has revealed that a substantial proportion of PSD-95 molecules (at least when overexpressed) is mobile within the synapse (Chazeau et al., 2014). As a preliminary attempt to investigate the role of a dynamic PSD, we explored in several models the potential effects of these various forms of PSD molecular dynamics. As expected, if PSD-95 scaffold molecules were permitted to diffuse, a larger subset of receptors could escape compared with the static case because the pattern of PSD-95 drifted toward randomized (data not shown). Other models incorporating less diffusion or other forms of motion that recapitulated the observed maintenance of PSD-95 density patterns could produce large, moderate, or negligible effects. Thus, dynamics within the PSD can alter the relative contribution of steric effects on receptor retention. However, detailed effects of this morphological plasticity depend very strongly on parameters that have not yet been deduced experimentally. Therefore, it will be important to gain more quantitative understanding of PSD molecular dynamics to gauge the time course over which these dynamics permit escape of sterically trapped receptors, or the trapping of proteins newly incorporated to the PSD.

It is tempting to speculate that such synaptic scaffold protein dynamics may play an important role in functional synaptic plasticity. Supporting this notion, molecular destabilization of the PSD is prominent during induction of LTP and LTD (Steiner et al., 2008; Xu et al., 2008), suggesting that periods of plasticity involve enhanced reorganization or disassembly of internal scaffold distribution. These observations are consistent with the model that synaptic potentiation could result from reorganization and restabilization of scaffolds to favor retention of bulky membrane proteins. On the other hand, synaptic depression could arise from homogenization and mobilization of scaffold positions, favoring dispersion of receptors. Thus, identifying mechanisms that govern the positional dynamics of synaptic scaffolding proteins themselves will be crucial to understanding the synaptic modifications that underlie learning, memory, and neuropsychiatric disorder.

\section{References}

Arendt KL, Royo M, Fernández-Monreal M, Knafo S, Petrok CN, Martens JR, Esteban JA (2010) PIP3 controls synaptic function by maintaining AMPA receptor clustering at the postsynaptic membrane. Nat Neurosci 13:36-44. CrossRef Medline

Ashby MC, Maier SR, Nishimune A, Henley JM (2006) Lateral diffusion drives constitutive exchange of AMPA receptors at dendritic spines and is regulated by spine morphology. J Neurosci 26:7046-7055. CrossRef Medline

Bats C, Groc L, Choquet D (2007) The interaction between Stargazin and PSD-95 regulates AMPA receptor surface trafficking. Neuron 53:719734. CrossRef Medline

Biermann B, Sokoll S, Klueva J, Missler M, Wiegert JS, Sibarita JB, Heine M (2014) Imaging of molecular surface dynamics in brain slices using single-particle tracking. Nat Commun 5:3024. CrossRef Medline

Blanpied TA, Kerr JM, Ehlers MD (2008) Structural plasticity with preserved topology in the postsynaptic protein network. Proc Natl Acad Sci U S A 105:12587-12592. CrossRef Medline

Borgdorff AJ, Choquet D (2002) Regulation of AMPA receptor lateral movements. Nature 417:649-653. CrossRef Medline

Breillat C, Thoumine O, Choquet D (2007) Characterization of SynCAM surface trafficking using a SynCAM derived ligand with high homophilic binding affinity. Biochem Biophys Res Commun 359:655-659. CrossRef Medline

Burette AC, Lesperance T, Crum J, Martone M, Volkmann N, Ellisman MH, Weinberg RJ (2012) Electron tomographic analysis of synaptic ultrastructure. J Comp Neurol 520:2697-2711. CrossRef Medline

Chazeau A, Mehidi A, Nair D, Gautier JJ, Leduc C, Chamma I, Kage F, Kechkar A, Thoumine O, Rottner K, Choquet D, Gautreau A, Sibarita JB, Giannone G (2014) Nanoscale segregation of actin nucleation and elongation factors determines dendritic spine protrusion. EMBO J 33:27452764. CrossRef Medline

Chen L, Chetkovich DM, Petralia RS, Sweeney NT, Kawasaki Y, Wenthold RJ, Bredt DS, Nicoll RA (2000) Stargazin regulates synaptic targeting of AMPA receptors by two distinct mechanisms. Nature 408:936-943. CrossRef Medline

Chen X, Winters C, Azzam R, Li X, Galbraith JA, Leapman RD, Reese TS (2008) Organization of the core structure of the postsynaptic density. Proc Natl Acad Sci U S A 105:4453-4458. CrossRef Medline

Chen X, Nelson CD, Li X, Winters CA, Azzam R, Sousa AA, Leapman RD, Gainer H, Sheng M, Reese TS (2011) PSD-95 is required to sustain the molecular organization of the postsynaptic density. J Neurosci 31:6329_ 6338. CrossRef Medline

Cheng D, Hoogenraad CC, Rush J, Ramm E, Schlager MA, Duong DM, Xu P, Wijayawardana SR, Hanfelt J, Nakagawa T, Sheng M, Peng J (2006) Relative and absolute quantification of postsynaptic density proteome isolated from rat forebrain and cerebellum. Mol Cell Proteomics 5:1158 1170. CrossRef Medline

Chetkovich DM, Chen L, Stocker TJ, Nicoll RA, Bredt DS (2002) Phosphorylation of the postsynaptic density-95 (PSD-95)/discs large/zona occludens- 1 binding site of stargazin regulates binding to PSD-95 and synaptic targeting of AMPA receptors. J Neurosci 22:5791-5796. Medline

Cho CH, St-Gelais F, Zhang W, Tomita S, Howe JR (2007) Two families of TARP isoforms that have distinct effects on the kinetic properties of AMPA receptors and synaptic currents. Neuron 55:890-904. CrossRef Medline

Choi J, Ko J, Park E, Lee JR, Yoon J, Lim S, Kim E (2002) Phosphorylation of stargazin by protein kinase A regulates its interaction with PSD-95. J Biol Chem 277:12359-12363. CrossRef Medline

Choquet D, Triller A (2013) The dynamic synapse. Neuron 80:691-703. CrossRef Medline

Constals A, Penn AC, Compans B, Toulmé E, Phillipat A, Marais S, Retailleau N, Hafner AS, Coussen F, Hosy E, Choquet D (2015) Glutamateinduced AMPA receptor desensitization increases their mobility and modulates short-term plasticity through unbinding from Stargazin. Neuron 85:787-803. CrossRef Medline

Ehlers MD, Heine M, Groc L, Lee MC, Choquet D (2007) Diffusional trapping of GluR1 AMPA receptors by input-specific synaptic activity. Neuron 54:447-460. CrossRef Medline

Fortin DA, Tillo SE, Yang G, Rah JC, Melander JB, Bai S, Soler-Cedeño O, Qin M, Zemelman BV, Guo C, Mao T, Zhong H (2014) Live imaging of endogenous PSD-95 using ENABLED: a conditional strategy to fluores- 
cently label endogenous proteins. J Neurosci 34:16698-16712. CrossRef Medline

Franks KM, Stevens CF, Sejnowski TJ (2003) Independent sources of quantal variability at single glutamatergic synapses. J Neurosci 23:3186-3195. Medline

Frischknecht R, Heine M, Perrais D, Seidenbecher CI, Choquet D, Gundelfinger ED (2009) Brain extracellular matrix affects AMPA receptor lateral mobility and short-term synaptic plasticity. Nat Neurosci 12:897904. CrossRef Medline

Frost NA, Shroff H, Kong H, Betzig E, Blanpied TA (2010) Single-molecule discrimination of discrete perisynaptic and distributed sites of actin filament assembly within dendritic spines. Neuron 67:86-99. CrossRef Medline

Gerrow K, Triller A (2010) Synaptic stability and plasticity in a floating world. Curr Opin Neurobiol 20:631-639. CrossRef Medline

Giannone G, Hosy E, Levet F, Constals A, Schulze K, Sobolevsky AI, Rosconi MP, Gouaux E, Tampé R, Choquet D, Cognet L (2010) Dynamic superresolution imaging of endogenous proteins on living cells at ultra-high density. Biophys J 99:1303-1310. CrossRef Medline

Granger AJ, Shi Y, Lu W, Cerpas M, Nicoll RA (2013) LTP requires a reserve pool of glutamate receptors independent of subunit type. Nature 493: 495-500. CrossRef Medline

Gray NW, Weimer RM, Bureau I, Svoboda K (2006) Rapid redistribution of synaptic PSD-95 in the neocortex in vivo. PLoS Biol 4:e370. CrossRef Medline

Haselwandter CA, Calamai M, Kardar M, Triller A, da Silveira RA (2011) Formation and stability of synaptic receptor domains. Phys Rev Lett 106: 238104. CrossRef Medline

Hastie P, Ulbrich MH, Wang HL, Arant RJ, Lau AG, Zhang Z, Isacoff EY, Chen L (2013) AMPA receptor/TARP stoichiometry visualized by single-molecule subunit counting. Proc Natl Acad Sci U S A 110:51635168. CrossRef Medline

Heine M, Groc L, Frischknecht R, Béique JC, Lounis B, Rumbaugh G, Huganir RL, Cognet L, Choquet D (2008) Surface mobility of postsynaptic AMPARs tunes synaptic transmission. Science 320:201-205. CrossRef Medline

Holcman D, Triller A (2006) Modeling synaptic dynamics driven by receptor lateral diffusion. Biophys J 91:2405-2415. CrossRef Medline

Hoze N, Nair D, Hosy E, Sieben C, Manley S, Herrmann A, Sibarita JB, Choquet D, Holcman D (2012) Heterogeneity of AMPA receptor trafficking and molecular interactions revealed by superresolution analysis of live cell imaging. Proc Natl Acad Sci U S A 109:17052-17057. CrossRef Medline

Husi H, Ward MA, Choudhary JS, Blackstock WP, Grant SG (2000) Proteomic analysis of NMDA receptor-adhesion protein signaling complexes. Nat Neurosci 3:661-669. CrossRef Medline

Kalashnikova E, Lorca RA, Kaur I, Barisone GA, Li B, Ishimaru T, Trimmer JS, Mohapatra DP, Díaz E (2010) SynDIG1: an activity-regulated, AMPA- receptor-interacting transmembrane protein that regulates excitatory synapse development. Neuron 65:80-93. CrossRef Medline

Kerr JM, Blanpied TA (2012) Subsynaptic AMPA receptor distribution is acutely regulated by actin-driven reorganization of the postsynaptic density. J Neurosci 32:658-673. CrossRef Medline

Kessels HW, Malinow R (2009) Synaptic AMPA receptor plasticity and behavior. Neuron 61:340-350. CrossRef Medline

Kohn JE, Millett IS, Jacob J, Zagrovic B, Dillon TM, Cingel N, Dothager RS, Seifert S, Thiyagarajan P, Sosnick TR, Hasan MZ, Pande VS, Ruczinski I, Doniach S, Plaxco KW (2004) Random-coil behavior and the dimensions of chemically unfolded proteins. Proc Natl Acad Sci U S A 101: 12491-12496. CrossRef Medline

Kucik DF, Elson EL, Sheetz MP (1999) Weak dependence of mobility of membrane protein aggregates on aggregate size supports a viscous model of retardation of diffusion. Biophys J 76:314-322. CrossRef Medline

Kuriu T, Inoue A, Bito H, Sobue K, Okabe S (2006) Differential control of postsynaptic density scaffolds via actin-dependent and -independent mechanisms. J Neurosci 26:7693-7706. CrossRef Medline

Levet F, Hosy E, Kechkar A, Butler C, Beghin A, Choquet D, Sibarita JB (2015) SR-Tesseler: a method to segment and quantify localizationbased super-resolution microscopy data. Nat Methods 12:1065-1071. CrossRef Medline

Lisman JE, Raghavachari S, Tsien RW (2007) The sequence of events that underlie quantal transmission at central glutamatergic synapses. Nat Rev Neurosci 8:597-609. CrossRef Medline

Lu HE, MacGillavry HD, Frost NA, Blanpied TA (2014) Multiple spatial and kinetic subpopulations of CaMKII in spines and dendrites as resolved by single-molecule tracking PALM. J Neurosci 34:7600-7610. CrossRef Medline

MacGillavry HD, Song Y, Raghavachari S, Blanpied TA (2013) Nanoscale scaffolding domains within the postsynaptic density concentrate synaptic AMPA receptors. Neuron 78:615-622. CrossRef Medline

Masson JB, Dionne P, Salvatico C, Renner M, Specht CG, Triller A, Dahan M (2014) Mapping the energy and diffusion landscapes of membrane proteins at the cell surface using high-density single-molecule imaging and Bayesian inference: application to the multiscale dynamics of glycine receptors in the neuronal membrane. Biophys J 106:74-83. CrossRef Medline

Matsuzaki M, Ellis-Davies GC, Nemoto T, Miyashita Y, Iino M, Kasai H (2001) Dendritic spine geometry is critical for AMPA receptor expression in hippocampal CA1 pyramidal neurons. Nat Neurosci 4:10861092. CrossRef Medline

Meier J, Vannier C, Sergé A, Triller A, Choquet D (2001) Fast and reversible trapping of surface glycine receptors by gephyrin. Nat Neurosci 4:253260. CrossRef Medline

Milstein AD, Zhou W, Karimzadegan S, Bredt DS, Nicoll RA (2007) TARP subtypes differentially and dose-dependently control synaptic AMPA receptor gating. Neuron 55:905-918. CrossRef Medline

Nair D, Hosy E, Petersen JD, Constals A, Giannone G, Choquet D, Sibarita JB (2013) Super-resolution imaging reveals that AMPA receptors inside synapses are dynamically organized in nanodomains regulated by PSD95. J Neurosci 33:13204-13224. CrossRef Medline

Nakagawa T, Cheng Y, Ramm E, Sheng M, Walz T (2005) Structure and different conformational states of native AMPA receptor complexes. Nature 433:545-549. CrossRef Medline

Opazo P, Choquet D (2011) A three-step model for the synaptic recruitment of AMPA receptors. Mol Cell Neurosci 46:1-8. CrossRef Medline

Park M, Salgado JM, Ostroff L, Helton TD, Robinson CG, Harris KM, Ehlers MD (2006) Plasticity-induced growth of dendritic spines by exocytic trafficking from recycling endosomes. Neuron 52:817-830. CrossRef Medline

Raghavachari S, Lisman JE (2004) Properties of quantal transmission at CA1 synapses. J Neurophysiol 92:2456-2467. CrossRef Medline

Renner ML, Cognet L, Lounis B, Triller A, Choquet D (2009b) The excitatory postsynaptic density is a size exclusion diffusion environment. Neuropharmacology 56:30-36. CrossRef Medline

Renner M, Choquet D, Triller A (2009a) Control of the postsynaptic membrane viscosity. J Neurosci 29:2926-2937. CrossRef Medline

Saffman PG, Delbrück M (1975) Brownian motion in biological membranes. Proc Natl Acad Sci U S A 72:3111-3113. CrossRef Medline

Sainlos M, Tigaret C, Poujol C, Olivier NB, Bard L, Breillat C, Thiolon K, Choquet D, Imperiali B (2011) Biomimetic divalent ligands for the acute disruption of synaptic AMPAR stabilization. Nat Chem Biol 7: 81-91. CrossRef Medline

Santamaria F, Gonzalez J, Augustine GJ, Raghavachari S (2010) Quantifying the effects of elastic collisions and non-covalent binding on glutamate receptor trafficking in the post-synaptic density. PLoS Comp Biol 6:e1000780. CrossRef Medline

Saxton MJ (2010) Two-dimensional continuum percolation threshold for diffusing particles of nonzero radius. Biophys J 99:1490-1499. CrossRef Medline

Schauder DM, Kuybeda O, Zhang J, Klymko K, Bartesaghi A, Borgnia MJ, Mayer ML, Subramaniam S (2013) Glutamate receptor desensitization is mediated by changes in quaternary structure of the ligand binding domain. Proc Natl Acad Sci U S A 110:5921-5926. CrossRef Medline

Schneider R, Hosy E, Kohl J, Klueva J, Choquet D, Thomas U, Voigt A, Heine M (2015) Mobility of calcium channels in the presynaptic membrane. Neuron 86:672-679. CrossRef Medline

Schwenk J, Harmel N, Zolles G, Bildl W, Kulik A, Heimrich B, Chisaka O, Jonas P, Schulte U, Fakler B, Klöcker N (2009) Functional proteomics identify cornichon proteins as auxiliary subunits of AMPA receptors. Science 323:1313-1319. CrossRef Medline

Schwenk J, Harmel N, Brechet A, Zolles G, Berkefeld H, Müller CS, Bildl W, Baehrens D, Hüber B, Kulik A, Klöcker N, Schulte U, Fakler B (2012) High-resolution proteomics unravel architecture and molecular diversity 
of native AMPA receptor complexes. Neuron 74:621-633. CrossRef Medline

Sergé A, Fourgeaud L, Hemar A, Choquet D (2002) Receptor activation and homer differentially control the lateral mobility of metabotropic glutamate receptor 5 in the neuronal membrane. J Neurosci 22:3910-3920. Medline

Sharma K, Fong DK, Craig AM (2006) Postsynaptic protein mobility in dendritic spines: long-term regulation by synaptic NMDA receptor activation. Mol Cell Neurosci 31:702-712. CrossRef Medline

Sheng M, Hoogenraad CC (2007) The postsynaptic architecture of excitatory synapses: a more quantitative view. Annu Rev Biochem 76:823-847. CrossRef Medline

Sheng M, Kim E (2011) The postsynaptic organization of synapses. Cold Spring Harb Perspect Biol 3:piia005678. CrossRef Medline

Simon CM, Hepburn I, Chen W, De Schutter E (2014) The role of dendritic spine morphology in the compartmentalization and delivery of surface receptors. J Comput Neurosci 36:483-497. CrossRef Medline

Soto D, Coombs ID, Renzi M, Zonouzi M, Farrant M, Cull-Candy SG (2009) Selective regulation of long-form calcium-permeable AMPA receptors by an atypical TARP, gamma-5. Nat Neurosci 12:277-285. CrossRef Medline

Steiner P, Higley MJ, Xu W, Czervionke BL, Malenka RC, Sabatini BL (2008) Destabilization of the postsynaptic density by PSD-95 serine 73 phosphorylation inhibits spine growth and synaptic plasticity. Neuron 60: 788-802. CrossRef Medline

Tomita S, Chen L, Kawasaki Y, Petralia RS, Wenthold RJ, Nicoll RA, Bredt DS (2003) Functional studies and distribution define a family of transmem- brane AMPA receptor regulatory proteins. J Cell Biol 161:805-816. CrossRef Medline

Tomita S, Fukata M, Nicoll RA, Bredt DS (2004) Dynamic interaction of stargazin-like TARPs with cycling AMPA receptors at synapses. Science 303:1508-1511. CrossRef Medline

Trimble WS, Grinstein S (2015) Barriers to the free diffusion of proteins and lipids in the plasma membrane. J Cell Biol 208:259-271. CrossRef Medline

von Engelhardt J, Mack V, Sprengel R, Kavenstock N, Li KW, Stern-Bach Y, Smit AB, Seeburg PH, Monyer H (2010) CKAMP44: a brain-specific protein attenuating short-term synaptic plasticity in the dentate gyrus. Science 327:1518-1522. CrossRef Medline

Xie X, Liaw JS, Baudry M, Berger TW (1997) Novel expression mechanism for synaptic potentiation: alignment of presynaptic release site and postsynaptic receptor. Proc Natl Acad Sci U S A 94:6983-6988. CrossRef Medline

Xu W, Schlüter OM, Steiner P, Czervionke BL, Sabatini B, Malenka RC (2008) Molecular dissociation of the role of PSD-95 in regulating synaptic strength and LTD. Neuron 57:248-262. CrossRef Medline

Zhang M, Chang H, Zhang Y, Yu J, Wu L, Ji W, Chen J, Liu B, Lu J, Liu Y, Zhang J, Xu P, Xu T (2012) Rational design of true monomeric and bright photoactivatable fluorescent proteins. Nat Methods 9:727-729. CrossRef Medline

Ziv NE, Fisher-Lavie A (2014) Presynaptic and postsynaptic scaffolds: dynamics fast and slow. Neuroscientist 20:439-452. CrossRef Medline 